\title{
Elementary abelian regular coverings of Platonic maps
}

\section{Case I: ordinary representations}

\author{
Gareth A. Jones
}

Received: 22 April 2012 / Accepted: 25 June 2014 / Published online: 17 July 2014

(C) Springer Science+Business Media New York 2014

\begin{abstract}
We classify those orientably regular maps which are elementary abelian regular branched coverings of Platonic maps $\mathcal{M}$, in the case where the covering group and the rotation group $G$ of $\mathcal{M}$ have coprime orders. The method involves studying the representations of $G$ on certain homology groups of the sphere, punctured at the branch-points. We give a complete classification for branching over faces (or, dually, vertices) of $\mathcal{M}$, and we outline how the method extends to other branching patterns.
\end{abstract}

Keywords Orientably regular map · Platonic map · Elementary abelian covering . Automorphism group

Mathematics Subject Classification $\quad 05 \mathrm{C} 10 \cdot 20 \mathrm{~B} 25$

\section{Introduction}

This paper addresses the problem of classifying orientably regular maps, those maps $\mathcal{N}$ on surfaces (always assumed to be compact and orientable) for which the orientationpreserving automorphism group $\mathrm{Aut}^{+} \mathcal{N}$ acts transitively on directed edges. Such maps are often formed as regular coverings of a simpler orientably regular map $\mathcal{M}$, in which case they correspond to normal subgroups of the fundamental group $\pi_{1} \mathcal{S}$ of the underlying surface $\mathcal{S}$ of $\mathcal{M}$, punctured at any branch-points, which are invariant under the induced action of the orientation-preserving automorphism group $G:=$ Aut $^{+} \mathcal{M}$ of $\mathcal{M}$ on $\pi_{1} \mathcal{S}$. In the case of abelian coverings, one can abelianise $\pi_{1} \mathcal{S}$, and instead look for $G$-invariant submodules of the first homology group $H_{1}(\mathcal{S} ; \mathbb{Z})$ : for instance,

G. A. Jones $(\varangle)$

School of Mathematics, University of Southampton, Southampton SO17 1BJ, UK

e-mail: g.a.jones@maths.soton.ac.uk 
Surowski and Jones [13] have used this method to classify the cyclic regular coverings of the Platonic maps $\mathcal{M}$, branched over the vertices, edges or faces; this work has recently been extended by $\mathrm{Hu}$ [9] to include branching over vertices and faces (see also [10]). When considering coverings by elementary abelian $p$-groups, one can go further and reduce the homology module $\bmod p$, so that $G$ acts on the vector space $H_{1}\left(\mathcal{S} ; \mathbb{F}_{p}\right)$ : this idea has been used by Kazaz [14-16] to classify the elementary abelian unbranched regular coverings of the orientably regular hypermaps of genus 2 .

Here we combine these two approaches to study regular branched coverings of the Platonic maps, the orientably regular maps of genus 0 , by elementary abelian $p$-groups, as a first step towards a more general classification of the abelian coverings of these maps (indeed, it is hoped that the techniques used here can also be applied to maps on other surfaces than the sphere). The representation theory of the corresponding Platonic groups $G$ plays an important role in this work. It is rather easier to apply when the prime $p$ does not divide $|G|$, as we shall generally assume here, so that Maschke's Theorem applies, and ordinary representation theory can be used. The remaining cases, where $p$ divides $|G|$ and modular representation theory is required, will be considered in a separate paper.

The action of automorphism groups on homology has been used, either explicitly or implicitly, to study abelian coverings in other contexts. For instance, it is a classical technique for Riemann surfaces, with Farkas and Kra [7, Sect. V.3] describing the background theory and Cohen [2] applying it to Klein's quartic curve. In recent years it has also been applied, often using the language of voltage assignments, to abelian coverings of graphs. For instance Malnič et al. [19] have developed the theory and applied it to elementary abelian coverings of dipoles and of the Heawood graph, while Kwak and Oh [17] and Conder and Ma [4,5] have respectively considered elementary abelian coverings of the octahedral graph and abelian coverings of various cubic graphs. In fact, the present work could be restated in terms of coverings of the Platonic graphs, restricted to those coverings which respect the surface-embeddings.

The main result is the following theorem, which uses the notation $\{n, m\}$ of Coxeter and Moser [6] for the $m$-valent Platonic map with $n$-gonal faces:

Theorem 1 The orientably regular covering maps $\mathcal{N}$ of the Platonic maps $\mathcal{M}$, branched over the faces, with elementary abelian p-groups of rank $c \geq 1$ as covering groups for primes $p$ not dividing the order of the group $G=$ Aut $^{+} \mathcal{M}$, are (up to orientation-preserving isomorphism) as follows:

- if $\mathcal{M}$ is the tetrahedron $\{3,3\}$ there is one map $\mathcal{N}$ for each $p>3$, with $c=3$;

- if $\mathcal{M}$ is the cube $\{4,3\}$ there are three maps $\mathcal{N}$ for each $p>3$, with $c=2,3$ and 5 ;

- if $\mathcal{M}$ is the octahedron $\{3,4\}$ there are seven maps $\mathcal{N}$ for each $p>3$, with $c=1,3,3,4,4,6$ and 7 ;

- if $\mathcal{M}$ is the dodecahedron $\{5,3\}$ there are seven maps $\mathcal{N}$ for each $p \equiv \pm 1(\bmod 5)$, with $c=3,3,5,6,8,8$ and 11 , and three when $3<p \equiv \pm 2(\bmod 5)$, with $c=5,6$ and 11 ; 
- if $\mathcal{M}$ is the icosahedron $\{3,5\}$ there are $8 p+23$ maps $\mathcal{N}$ for each $p \equiv \pm 1$ $(\bmod 5)$, with $c \in\{3,4, \ldots, 16,19\}$, and $4 p+11$ when $3<p \equiv \pm 2(\bmod 5)$, with $c \in\{4,5,6,8,9,10,11,13,14,15,19\}$;

- if $\mathcal{M}$ is the dihedron $\{n, 2\}$ there is one map $\mathcal{N}$ for each $p$ coprime to $2 n$, with $c=1$;

- if $\mathcal{M}$ is the hosohedron $\{2, n\}$ there are $2^{v}-1$ maps $\mathcal{N}$ for each $p$ coprime to $2 n$, where $v$ is the number of orbits $\Delta \neq\{1\}$ of the group generated by the Frobenius automorphism and inversion on the set of $n$-th roots of 1 in the algebraic closure $\overline{\mathbb{F}}_{p}$ of the field $\mathbb{F}_{p}$.

For each $\mathcal{M}=\{n, m\}$ and prime $p$ these maps $\mathcal{N}$ have type $\{n p, m\}$ and genus

$$
\left(\frac{f}{2}-1\right) p^{c}-\frac{f}{2} p^{c-1}+1
$$

where $f$ is the number of faces of $\mathcal{M}$; they are all quotients of a single covering map with $c=f-1$. The maps $\mathcal{N}$ are all regular, except when $\mathcal{M}$ is the icosahedron, in which case there are $4(p-1)$ or $2(p-1)$ chiral pairs as $p \equiv \pm 1$ or $\pm 2(\bmod 5)$, the other 31 or 15 being regular. In all cases except when $\mathcal{M}$ is the icosahedron, the $2^{v}-1$ maps $\mathcal{N}$ are the joins formed from a set of $v$ irreducible coverings of $\mathcal{M}$.

\section{Comments}

(1) The orientation-preserving automorphism group $\mathrm{Aut}^{+} \mathcal{N}$ of each of these maps is an extension of the covering group, an elementary abelian normal subgroup $K$ of order $p^{c}$, by $G$. Since $G$ and $K$ have coprime orders, the Schur-Zassenhaus Theorem implies that this extension splits. As a $G$-module under conjugation, $K$ is isomorphic to a quotient of $H_{1}\left(S ; \mathbb{F}_{p}\right)$, identified specifically in Sects. 3-9 for the individual maps $\mathcal{M}$.

(2) An orientably regular map is called regular or chiral as it is or is not isomorphic to its mirror image. When $\mathcal{M}$ is the icosahedron, the chiral covering maps arise from the fact that its full automorphism group Aut $\mathcal{M}$ has fewer orbits than $G$ on ordered pairs of faces of $\mathcal{M}$ (see Sects. 2.6 and 7). The fact that in this case the number of coverings is unbounded as a function of $p$ is related to the fact that the permutation character of $G$ on faces of $\mathcal{M}$ is not multiplicity-free (see Sects. 2.5 and 7).

(3) In the case of the hosohedron, the method for finding the ranks $c$ of the covering groups $K$, too complicated to state here, is described in Sect. 9.2.

(4) The conclusions of Theorem 1 also apply in a few cases where $p$ divides $|G|$, such as the tetrahedron and octahedron with $p=3$, and the hosohedron with $n$ odd and $p=2$; these cases will be studied in a later paper.

The paper is organised as follows. In Sect. 2 some useful techniques are outlined. These are applied in Sects. 3-9 to enumerate and describe the coverings in Theorem 1 . The individual cases are fairly straightforward, except for that involving the hosohedron $\mathcal{M}=\{n, 2\}$, which depends on the reduction mod $p$ of cyclotomic polynomials. Branching over vertices is easily dealt with by duality. In Sect. 10 we consider 
simultaneous branching over vertices and faces, concentrating on the tetrahedron as a typical example. In Sects. 11 and 12 we briefly consider the hypermaps arising from branching over edges, and over vertices, edges and faces. The results for these different branching patterns are similar to those in Theorem 1.

\section{Techniques}

In this section, several techniques from algebraic map theory, topology and representation theory are used to reduce the classification problem for maps to a number of mainly group- and number-theoretic calculations. No originality is claimed for the results quoted, which are all standard in their areas. To help the reader through what is a rather long narrative, some important observations, often used implicitly in later calculations, are italicised.

\subsection{Maps}

First, we briefly sketch the connections between maps and triangle groups; for background, see [11]. Maps are always assumed to be oriented, that is, on orientable surfaces with a chosen orientation. The aim is to classify them up to orientation-preserving isomorphism.

We define a triangle group to be a group of the form

$$
\Delta(p, q, r)=\left\langle x, y, z \mid x^{p}=y^{q}=z^{r}=x y z=1\right\rangle
$$

where $p, q, r \in \mathbb{N} \cup\{\infty\}$ and we ignore any relation $g^{\infty}=1$. Any $m$-valent map $\mathcal{N}$ corresponds to a subgroup $N$ of the triangle group

$$
\Delta:=\Delta(m, 2, \infty)=\left\langle x, y, z \mid x^{m}=y^{2}=x y z=1\right\rangle,
$$

with vertices, edges and faces corresponding to the cycles of $x, y$ and $z$ on the cosets of $N$.

The map $\mathcal{N}$ is orientably regular if and only if $N$ is normal in $\Delta$, in which case the orientation-preserving automorphism group $\mathrm{Aut}^{+} \mathcal{N}$ is isomorphic to $\Delta / N$; we will assume this throughout.

In particular, the Platonic map $\mathcal{M}=\{n, m\}$ corresponds to the normal closure $M$ of $z^{n}$ in $\Delta$, and

$$
\mathrm{Aut}^{+} \mathcal{M} \cong \Delta / M \cong \Delta(m, 2, n) .
$$

A map $\mathcal{N}$ is a $d$-sheeted covering of $\mathcal{M}$ if and only if $N$ is a subgroup of index $d$ in $M$, in which case $\mathcal{N} \rightarrow \mathcal{M}$ is a regular covering and the group of covering transformations is $M / N$.

Thus the orientably regular $m$-valent maps which are $d$-sheeted abelian coverings of $\mathcal{M}$ are (up to orientation-preserving isomorphism) in bijective correspondence with the subgroups $N$ of $M$ which are normal in $\Delta$, with $M / N$ abelian of order $d$. 
If $M / N$ has exponent $e$, such subgroups $N$ contain the commutator subgroup $M^{\prime}$ and the subgroup $M^{e}$ generated by the $e$-th powers in $M$, so they correspond to subgroups $\bar{N}=N / M^{\prime} M^{e}$ of $\bar{M}=M / M^{\prime} M^{e}$, with $M / N \cong \bar{M} / \bar{N}$. The action of $\Delta$ by conjugation on the normal subgroup $M$ preserves its characteristic subgroups $M^{\prime}$ and $M^{e}$, giving an induced action of $\Delta$ on $\bar{M}$; since $M$ acts trivially on $\bar{M}$, we have an action of the group

$$
G:=\text { Aut }^{+} \mathcal{M} \cong \Delta / M
$$

on $\bar{M}$, which is a module for $G$ over the ring $\mathbb{Z}_{e}:=\mathbb{Z} / e \mathbb{Z}$.

It follows that a subgroup $N$ of $M$, containing $M^{\prime} M^{e}$, is normal in $\Delta$ if and only if $\bar{N}$ is a $G$-submodule of $\bar{M}$.

The orientation-preserving automorphism group $\tilde{G}:=$ Aut $^{+} \mathcal{N} \cong \Delta / N$ of $\mathcal{N}$ has an abelian normal subgroup $K$, corresponding to $M / N$, with $\tilde{G} / K \cong G$; under the induced action of $G$ on $K$ by conjugation, $K$ is isomorphic as a $G$-module to $\bar{M} / \bar{N}$. If $e$ is coprime to $|G|$, then the Schur-Zassenhaus Theorem implies that $\tilde{G}$ is a semidirect product of $K$ by $G$.

\subsection{Homology modules}

Let $S=S^{2} \backslash\left\{c_{1}, \ldots, c_{f}\right\}$, where $c_{1}, \ldots, c_{f}$ are the centres of the $f$ faces of $\mathcal{M}$. Then $M$ can be identified with the fundamental group $\pi_{1}(S)$ of $S$, a free group of rank $f-1$ generated by the homotopy classes $g_{i}$ of loops around the punctures $c_{i}$, with a single defining relation $g_{1} \ldots g_{f}=1$. It follows that the group $M^{\text {ab }}=M / M^{\prime}$ can be identified with the first integer homology group $H_{1}(S ; \mathbb{Z})=\pi_{1}(S)^{\text {ab }}$ of $S$, a free abelian group of rank $f-1$ generated by the homology classes $\left[g_{i}\right]$, with defining relation $\left[g_{1}\right]+\cdots+\left[g_{f}\right]=0$. By the Universal Coefficient Theorem, $\bar{M}=M / M^{\prime} M^{e}$ is identified with the $\bmod e$ homology group $H_{1}\left(S ; \mathbb{Z}_{e}\right)=H_{1}(S ; \mathbb{Z}) \otimes_{\mathbb{Z}} \mathbb{Z}_{e} \cong \mathbb{Z}_{e}^{f-1}$.

Under these identifications, the actions of $G$ induced by conjugation in $\Delta$ and by homeomorphisms of $S$ are the same, so our problem is to find the G-submodules of $H_{1}\left(S ; \mathbb{Z}_{e}\right)$.

The prime power factorisation $\prod_{p} p^{i_{p}}\left(p\right.$ prime, $i_{p} \geq 1$ ) of $e$ induces a $G$-invariant decomposition of $H_{1}\left(S ; \mathbb{Z}_{e}\right)$ as a direct sum of its Sylow $p$-subgroups $H_{1}\left(S ; \mathbb{Z}_{p^{i p}}\right)$, so it is sufficient to restrict attention to the case where $e$ is a power of a prime $p$. For each prime $p$ we have an infinite descending series

$$
M^{\mathrm{ab}}=M / M^{\prime}>M^{\prime} M^{p} / M^{\prime}>M^{\prime} M^{p^{2}} / M^{\prime}>\cdots>M^{\prime} M^{p^{i}} / M^{\prime}>\cdots,
$$

of characteristic subgroups of $M^{\mathrm{ab}}$, corresponding to a descending series

$$
H_{1}(S ; \mathbb{Z})=H^{0}>H^{1}>\cdots>H^{i}>\cdots,
$$


of $G$-submodules of $H_{1}(S ; \mathbb{Z})$, where each $H^{i}$ is the kernel of the reduction $\left(\bmod p^{i}\right): H_{1}(S ; \mathbb{Z}) \rightarrow H_{1}\left(S ; \mathbb{Z}_{p^{i}}\right)$. This induces a finite descending series

$$
H_{1}\left(S ; \mathbb{Z}_{p^{i}}\right)=H^{0} / H^{i}>H^{1} / H^{i}>\cdots>H^{i} / H^{i}=0
$$

of $G$-submodules of $H_{1}\left(S ; \mathbb{Z}_{p^{i}}\right)$. Successive quotients

$$
\left(H^{j} / H^{i}\right) /\left(H^{j+1} / H^{i}\right) \cong H^{j} / H^{j+1} \cong M^{\prime} M^{p^{j}} / M^{\prime} M^{p^{j+1}}
$$

in this series are $G$-isomorphic to the module

$$
H_{1}\left(S ; \mathbb{F}_{p}\right)=H_{1}\left(S ; \mathbb{Z}_{p}\right)=H^{0} / H^{1} \cong M_{p}:=M / M^{\prime} M^{p},
$$

where $\mathbb{F}_{p}$ is the field of $p$ elements. This $G$-module $H_{1}\left(S ; \mathbb{F}_{p}\right)$, along with all the other quotients in the series, is a vector space over $\mathbb{F}_{p}$ of dimension $f-1$ affording a representation $\rho_{p}: G \rightarrow \operatorname{Aut}\left(H_{1}\left(S ; \mathbb{F}_{p}\right)\right) \cong \mathrm{GL}_{f-1}\left(\mathbb{F}_{p}\right)$ of $G$.

Our main problem therefore is to determine the structure of this $G$-module $H_{1}\left(S ; \mathbb{F}_{p}\right)$ for each prime $p$; in particular, this will immediately determine the elementary abelian regular coverings of $\mathcal{M}$.

A $G$-submodule $L$ of $H_{1}\left(S ; \mathbb{F}_{p}\right)$ of codimension $c$ corresponds to a normal subgroup $N$ of $\Delta$ contained in $M$, with the group $K:=M / N$ elementary abelian of order $p^{c}$, and hence to a regular $p^{c}$-sheeted covering $\mathcal{N}$ of $\mathcal{M}$, branched over its face-centres, with covering group $K$. The image in $K$ of a generator $g_{i}$ of $M$ in $K$ is called a monodromy generator of $K$, representing the permutation of the sheets of the covering induced by traversing a small loop in $S$ around the corresponding branch-point.

This covering $\mathcal{N}$ is an orientably regular map of type $\{n p, m\}$. There are $p^{c-1}$ points above each of the $f$ face-centres of $\mathcal{M}$, so the total order of branching of the covering $\mathcal{N} \rightarrow \mathcal{M}$ is $\left(p^{c}-p^{c-1}\right) f$. By the Riemann-Hurwitz formula, $\mathcal{N}$ therefore has genus

$$
g=1-p^{c}+\frac{1}{2} p^{c-1}(p-1) f=\left(\frac{f}{2}-1\right) p^{c}-\frac{f}{2} p^{c-1}+1 .
$$

The orientation-preserving automorphism group $\tilde{G}=\operatorname{Aut}^{+} \mathcal{N}$ of $\mathcal{N}$ has an elementary abelian normal subgroup $K$ of order $p^{c}$, isomorphic to $H_{1}\left(S ; \mathbb{F}_{p}\right) / L$ as a $G$-module over $\mathbb{F}_{p}$.

For notational convenience, we let $E_{p}(\mathcal{M})$ denote the set of (orientation-preserving isomorphism classes of) such regular covers $\mathcal{N}$ of $\mathcal{M}$ with elementary abelian $p$ groups as covering groups, and for each $\mathcal{N} \in E_{p}(\mathcal{M})$ we let $\operatorname{dim}(\mathcal{N})=c$ where the covering $\mathcal{N} \rightarrow \mathcal{M}$ has $p^{c}$ sheets. Thus $\operatorname{dim}(\mathcal{N})$ is simply the dimension of the corresponding $G$-module $H_{1}\left(S ; \mathbb{F}_{p}\right) / L$, or equivalently the codimension of $L$ in $H_{1}\left(S ; \mathbb{F}_{p}\right)$. We will denote the representation of $G$ on $H_{1}\left(S ; \mathbb{F}_{p}\right) / L$ and the corresponding character by $\rho_{\mathcal{N}}$ and $\chi_{\mathcal{N}}$, and we will say that they are both afforded by $\mathcal{N}$; this is because $H_{1}\left(S ; \mathbb{F}_{p}\right) / L$ is isomorphic, as a $G$-module, to $M / N$ and hence to $K$.

The representation $\rho_{\mathcal{N}}$ can therefore be regarded as the induced action of $G$, by conjugation, on the covering group $K$ associated with $\mathcal{N}$. 
If $p$ is coprime to $|G|$ then the representation theory of $G$ over fields of characteristic $p$ is essentially the same as that over fields of characteristic 0 (ordinary representation theory): for instance Maschke's Theorem applies in both cases. In this situation, the decomposition of $H_{1}\left(S ; \mathbb{F}_{p}\right)$ as a $G$-module can be obtained from that of the corresponding homomology module over $\mathbb{C}$ (or indeed any algebraically closed field of characteristic 0 , such as the field $\overline{\mathbb{Q}}$ of algebraic numbers). Moreover, if $p$ is coprime to $|G|$ then $\tilde{G}$ is a semidirect product of $K$ by $G$, by the Schur-Zassenhaus Theorem.

If, on the other hand, $p$ divides $|G|$ then this extension splits if and only if its Sylow $p$-subgroups split over $K$. Moreover, in this situation, Maschke's Theorem does not apply, and the submodule structure of $H_{1}\left(S ; \mathbb{F}_{p}\right)$ is less transparent, requiring modular rather than ordinary representation theory. In this paper we therefore concentrate on the ordinary case, avoiding the finitely many primes $p$ dividing $|G|$; they will be considered in a separate paper.

\subsection{Permutation modules}

Let $\Phi$ be the set of faces of $\mathcal{M}$, and let $\mathbb{C} \Phi$ be the corresponding permutation module for $G$; this is an $f$-dimensional complex vector space with basis $\Phi$ permuted naturally by $G$. The homology module $H_{1}(S ; \mathbb{C})$ is isomorphic to the quotient of $\mathbb{C} \Phi$ by the 1-dimensional $G$-submodule spanned by the element $\sum_{\phi \in \Phi} \phi$.

The $G$-module decomposition of $\mathbb{C} \Phi$ corresponds to that of the corresponding permutation character $\pi$, where $\pi(g)$ is the number of faces $\phi \in \Phi$ invariant under each $g \in G$.

If $H$ denotes the subgroup $\langle z\rangle \cong C_{n}$ of $G$ leaving invariant a face, then since $G$ acts transitively on $\Phi$ we have $\pi=1_{H}^{G}$, the character obtained by inducing the principal character $1_{H}$ of $H$ up to $G$. By Frobenius reciprocity, the multiplicity $\left(\chi_{i}, \pi\right)$ of any irreducible complex character $\chi_{i}$ of $G$ in $\pi$ is given by

$$
\left(\chi_{i}, \pi\right)=\left(\left.\chi_{i}\right|_{H}, 1_{H}\right)=|H|^{-1} \sum_{g \in H} \chi_{i}(g),
$$

the multiplicity of $1_{H}$ in the restriction of $\chi_{i}$ to $H$.

Equivalently, the multiplicity $\left(\chi_{i}, \pi\right)$ of an irreducible character $\chi_{i}$ of $G$ in $\pi$ is the multiplicity of 1 as an eigenvalue of $z$ in the representation $\rho_{i}$ of $G$ corresponding to $\chi_{i}$

Given these multiplicities, we know the direct sum decomposition of $\mathbb{C} \Phi$, and hence that of $H_{1}(S ; \mathbb{C})$. In particular, this module affords the character

$$
\chi=\pi-\chi_{1},
$$

where $\chi_{1}$ is the principal character $1_{G}$ of $G$, given by $\chi_{1}(g)=1$ for all $g \in G$.

The same decompositions apply if $\mathbb{C}$ is replaced with the algebraic closure $\overline{\mathbb{F}}_{p}$ of $\mathbb{F}_{p}$ for any prime $p$ not dividing $|G|$. To find the corresponding decompositions over $\mathbb{F}_{p}$, various algebraically conjugate summands must be merged to give summands realised 
over $\mathbb{F}_{p}$. For the various Platonic maps $\mathcal{M}$, how this happens depends on whether $p$ satisfies certain congruences.

\subsection{Finding submodules}

Some submodules $L$ of $H_{1}\left(S ; \mathbb{F}_{p}\right)$ arise naturally from the action of $G$ on $\Phi$, whether or not $p$ divides $|G|$. Let $P$ denote the permutation module $\mathbb{F}_{p} \Phi$, a $G$-module over $\mathbb{F}_{p}$. Given any subset $\Psi \subseteq \Phi$, let $\underline{\Psi}$ denote the element $\sum_{\phi \in \Psi} \phi$ of $P$. The $G$ module $H_{1}\left(S ; \mathbb{F}_{p}\right)$ can then be identified with the quotient $Q:=P / P_{1}$ of $P$ by the 1-dimensional submodule $P_{1}$ spanned by $\underline{\Phi}$.

\subsubsection{The submodule $L=0$ of $Q$}

There is a regular covering $\mathcal{M}_{0} \in E_{p}(\mathcal{M})$, with $\operatorname{dim}\left(\mathcal{M}_{0}\right)=f-1$, corresponding to the submodule $L=0$ of $Q=H_{1}\left(S ; \mathbb{F}_{p}\right)$, i.e. to the submodule $P_{1}$ of $P$; all other regular coverings $\mathcal{N}$ of $\mathcal{M}$ in $E_{p}(\mathcal{M})$ are proper quotients of $\mathcal{M}_{0}$, so they satisfy $\operatorname{dim}(\mathcal{N}) \leq f-2$.

\subsubsection{The submodule $L=Q^{1}$ of $Q$}

There is a $G$-submodule $P^{1}$ of codimension 1 in $P$, consisting of the elements $\sum_{\phi \in \Phi} a_{\phi} \phi$ with coordinate-sum $\sum_{\phi \in \Phi} a_{\phi}=0$. If $p$ divides $f$ then $P_{1} \leq P^{1}$, giving a $G$-submodule $L=Q^{1}=P^{1} / P_{1}$ of codimension 1 in $Q$, and hence a regular covering $\mathcal{M}^{1} \in E_{p}(\mathcal{M})$ with $c=\operatorname{dim}\left(\mathcal{M}^{1}\right)=1$. This is therefore a cyclic covering of $\mathcal{M}$ (meaning that the covering group $K$ is cyclic), and since $L / Q^{1} \cong P / P^{1}$ affords the principal representation of $G$ it is also a central covering, in the sense that $K$ is in the centre of $\tilde{G}$. The cyclic coverings of the Platonic hypermaps were classified by the author and Surowski in [13] (see also [20]), where Proposition 3 implies that $\tilde{G}$ is a split extension of $G$ by $K$, giving $\tilde{G}=G \times K \cong G \times C_{p}$, if and only if $p$ divides $2 m$ but not $n$. In the cases where $p$ does not divide $f$ we have $P=P_{1} \oplus P^{1}$, so $Q \cong P^{1}$ and hence $P^{1}$ does not correspond to a proper covering of $\mathcal{M}$.

\subsubsection{The submodules $L=Q \sim$ of $Q$}

If $G$ acts imprimitively on $\Phi$, preserving a non-trivial equivalence relation (equivalently, if there is a subgroup $H_{\sim}$ of $G$ such that $H<H_{\sim}<G$ ), then as $\Psi$ ranges over the $k=\left|G: H_{\sim}\right|$ equivalence classes, the elements $\underline{\Psi}$ form a basis for a $k$-dimensional $G$-submodule $P_{\sim}$ of $P$, containing $P_{1}$ (and contained in $P^{1}$ if and only if $p$ divides the size $\left|H_{\sim}: H\right|=f / k$ of each class). The $G$-submodule $L=Q_{\sim}=P \sim / P_{1}$ of $Q$ therefore corresponds to a regular covering $\mathcal{M}_{\sim} \in E_{p}(\mathcal{M})$ of dimension $\operatorname{dim}\left(\mathcal{M}_{\sim}\right)=f-k$, affording the character $\chi_{\mathcal{M}}=\pi-\pi \sim$ of $G$ where $\pi_{\sim}$ is the permutation character of $G$ on the equivalence classes of $\sim$, i.e. on the cosets of $H_{\sim}$ in $G$. If $\approx$ is a $G$-invariant equivalence relation which refines $\sim$ (i.e. $\phi_{1} \approx \phi_{2}$ implies $\phi_{1} \sim \phi_{2}$ ), then the inclusion $Q \sim \leq Q \approx$ induces a covering $\mathcal{M}_{\sim} \rightarrow \mathcal{M} \approx$. 
For instance, suppose that $\mathcal{M}$ has an antipodal automorphism, so that $f$ is even. Then $P$ has a $G$-submodule $P_{a} \geq P_{1}$ of dimension $k=f / 2$ with basis elements $\underline{\Psi}$ corresponding to the antipodal pairs $\Psi \subseteq \Phi$. This gives a $G$-submodule $L=$ $Q_{a}=P_{a} / P_{1}$ of codimension $f-k=f / 2$ in $Q$, so we obtain a regular covering $\mathcal{M}_{a} \in E_{p}(\mathcal{M})$ with $\operatorname{dim}\left(\mathcal{M}_{a}\right)=f / 2$. If $p>2$ there is a second $G$-submodule $P_{a^{\prime}}$ of dimension $f / 2$ in $P$, with a basis element $\phi-\phi^{\prime}$ for each antipodal pair $\left\{\phi, \phi^{\prime}\right\} \subseteq \Phi$ (so $\left.P_{a^{\prime}} \leq P^{1}\right)$. The $G$-submodule $L=Q_{a^{\prime}}=\left(P_{a^{\prime}} \oplus P_{1}\right) / P_{1}$ of codimension $(f-2) / 2$ in $Q$ corresponds to a regular covering $\mathcal{M}_{a^{\prime}} \in E_{p}(\mathcal{M})$ with $\operatorname{dim}\left(\mathcal{M}_{a^{\prime}}\right)=(f-2) / 2$. A simple calculation shows that $P=P_{a} \oplus P_{a^{\prime}}$, so $Q=Q_{a} \oplus Q_{a^{\prime}}$. Since $P_{a}$ affords the permutation character $\pi_{a}$ of $G$ on the antipodal pairs of faces, it follows that $Q_{a}$ and hence $Q / Q_{a^{\prime}}$ afford $\pi_{a}-\chi_{1}$, while $Q_{a^{\prime}}$ and $Q / Q_{a}$ afford $\chi-\pi_{a}$.

\subsubsection{The case $p=2$}

If $p=2$ one can identify $P$ with the power set $\mathcal{P}(\Phi)$ of $\Phi$, a group under symmetric difference, with the natural induced action of $G$, so that $P^{1}=\{\Psi \subseteq \Phi|| \Psi \mid$ is even $\}$ and $P_{1}=\{\emptyset, \Phi\}$. In this case, $Q$ can be identified with the set of complementary pairs $\{\Psi, \Phi \backslash \Psi\}$ of subsets of $\Phi$.

\subsubsection{The natural representation $\rho_{n}$}

For each $\mathcal{M}$, the group $G$ has a natural 3-dimensional complex representation $\rho_{n}$, obtained by extending its natural real representation, as the rotation group of $\mathcal{M}$, by linearity to $\mathbb{C}$. This representation is irreducible, except when $\mathcal{M}$ is the dihedron or hosohedron, in which case it splits as a direct sum of 1- and 2-dimensional representations. When $\mathcal{M}$ is one of the five Platonic solids, by sending each basis element $\phi(\phi \in \Phi)$ of $\mathbb{C} \Phi$ to the position-vector in $\mathbb{R}^{3} \subset \mathbb{C}^{3}$ of the face-centre of $\phi$, we see that $\rho_{n}$ is a quotient, and hence a direct summand, of the representation of $G$ on $\mathbb{C} \Phi$. Frobenius reciprocity implies that $\rho_{n}$ has multiplicity 1 in this representation, and in the cases of the dodecahedron and icosahedron, the same applies to the Galois conjugate representation $\rho_{n *}$. By realising $\rho_{n}$ over the ring of integers of an appropriate algebraic number field (namely $\mathbb{Q}$ for the tetrahedron, cube or octahedron, $\mathbb{Q}(\sqrt{5})$ for the dodecahedron or icosahedron, and a cyclotomic field $\mathbb{Q}\left(\zeta_{n}\right)$ for the dihedron or hosohedron, where $\left.\zeta_{n}=e^{2 \pi i / n}\right)$, and taking quotients modulo some prime ideal containing $p$, we find that the $\bmod p$ reduction of $\rho_{n}$ or of $\rho_{n} \oplus \rho_{n *}$ is a summand of the representation of $G$ on $P$, and hence on $Q$.

\subsection{Diagonal submodules}

We need to deal with those cases where $Q$ has summands of multiplicity greater than one. If $A_{1}$ and $A_{2}$ are isomorphic $F G$-modules for some field $F$ and group $G$, then a diagonal submodule of $A_{1} \oplus A_{2}$ is a submodule of the form $\left\{\left(a, a^{\prime}\right) \mid a \in A_{1}\right\}$ for some isomorphism $A_{1} \rightarrow A_{2}, a \mapsto a^{\prime}$. 
Lemma 1 Let $U$ be a submodule of a module $V=V_{1} \oplus V_{2}$, let $U_{i}=U \cap V_{i}$, and let $\bar{V}_{i}=V_{i} / U_{i}$ for $i=1,2$. Then $U$ is the inverse image in $V$ of a diagonal submodule of $\bar{W}_{1} \oplus \bar{W}_{2}$ for some submodules $W_{i}$ such that $U_{i} \leq W_{i} \leq V_{i}$ for $i=1,2$.

Proof The image of $U$ in $\bar{V}_{1} \oplus \bar{V}_{2}$ intersects each summand $\bar{V}_{i}$ trivially, so it is isomorphic to its projection $\bar{W}_{i}$ in each $\bar{V}_{i}$, and is therefore a diagonal submodule of $\bar{W}_{1} \oplus \bar{W}_{2}$.

Thus, if the submodule structures of each $V_{i}$ are known, so is that of $V$.

Lemma 2 If $V_{1}$ and $V_{2}$ are isomorphic irreducible $F G$-modules, then the only proper submodules of $V_{1} \oplus V_{2}$ are $0, V_{1}, V_{2}$ and the diagonal submodules; given any isomorphism $V_{1} \rightarrow V_{2}, v \mapsto v^{\prime}$, each of the latter has the form $\left\{\left(v, \lambda v^{\prime}\right) \mid v \in V_{1}\right\}$ for some $\lambda \in F^{*}$.

Proof Let $U$ be a submodule of $V=V_{1} \oplus V_{2}$. In the notation of Lemma 1, for each $i=1,2$ the irreducibility of $V_{i}$ implies that $U_{i}=0$ or $V_{i}$. If $U_{i}=V_{i}$ for some $i$ then $U \geq V_{i}$, so the irreducibility of $V / V_{i}$ implies that $U=V_{i}$ or $V$. Otherwise, $U_{1}=U_{2}=0$, so in Lemma 1 we have either $W_{i}=0$ for some $i$, giving $U=0$, or $W_{i}=V_{i}$ for each $i$, in which case $U$ is a diagonal submodule of $V$. In the latter case, Schur's Lemma implies that the isomorphisms $V_{1} \rightarrow V_{2}$ are the functions $v \mapsto \lambda v^{\prime}$ for $\lambda \in F^{*}$.

This shows that if $V_{1}=V_{2}$ is irreducible and $F=\mathbb{F}_{q}$, then $V=V_{1} \oplus V_{2}=V_{1} \oplus V_{1}$ has $q+1$ non-trivial proper submodules, all isomorphic to $V_{1}$. We can write them as

$$
V(\lambda)=\left\{(\alpha v, \beta v) \mid v \in V_{1}, \alpha / \beta=\lambda\right\}
$$

for $\lambda \in \mathbb{P}^{1}\left(\mathbb{F}_{q}\right)=\mathbb{F}_{q} \cup\{\infty\}$; here $V(\infty)$ and $V(0)$ are the first and second direct summands, with $\beta=0$ and $\alpha=0$, respectively.

\subsection{Regularity and chirality}

Each of the covering maps $\mathcal{N}$ constructed here is orientably regular, since it corresponds to a normal subgroup $N$ of $\Delta$. It is regular (i.e. it also admits an orientationreversing automorphism) if and only if $N$ is normal in the extended triangle group $\Gamma=\Delta[m, 2, \infty]$, which contains $\Delta$ as a subgroup of index 2 . This is equivalent to the corresponding submodule $L$ of $H_{1}\left(S ; \mathbb{Z}_{e}\right)$ being invariant, not just under the orientation-preserving automorphism group $G=$ Aut $^{+} \mathcal{M} \cong \Delta / N$ of $\mathcal{M}$, but under its full automorphism group $A=$ Aut $\mathcal{M} \cong \Gamma / N$. When $e=p$, it is clear from their construction that the maps $\mathcal{M}_{0}, \mathcal{M}^{1}, \mathcal{M}_{a}$ and $\mathcal{M}_{a^{\prime}}$ (where they exist) are all regular.

One can find the structure of $H_{1}\left(S ; \mathbb{F}_{p}\right)$ as an A-module by treating it as the quotient $Q=P / P_{1}$ of $P$, where $P$ is now regarded as the permutation module for $A$ on $\Phi$.

In particular, the permutation character $\left.\pi\right|_{A}$ of $A$ on $P$ can be found as for $\left.\pi\right|_{G}$ (see Sect. 2.3), taking $H$ to be the stabiliser $A_{\phi} \cong D_{n}$ of a face $\phi \in \Phi$ in $A$ instead of its stabiliser $G_{\phi} \cong C_{n}$ in $G$. 
Lemma 3 If $\mathcal{M}$ is not the icosahedron, and if $p$ is a prime not dividing $|G|$, then all the maps $\mathcal{N} \in E_{p}(\mathcal{M})$ are regular.

Proof It is sufficient to show that the $G$-submodules of $H_{1}(S ; \mathbb{C})$ are all $A$-invariant, i.e. that the $A$ - and $G$-module direct sum decompositions of $H_{1}(S ; \mathbb{C})$ are identical. If this is not the case, then at least one irreducible summand of this $A$-module must split into a direct sum of two or more irreducible summands of the $G$-module, so that the permutation character $\pi$ on $\Phi$ satisfies $\left(\left.\pi\right|_{A},\left.\pi\right|_{A}\right)_{A}<\left(\left.\pi\right|_{G},\left.\pi\right|_{G}\right)_{G}$. But the two sides of this inequality are equal to the ranks of $A$ and $G$ as permutation groups on $\Phi$, i.e. the number of orbits of $A$ and $G$ on $\Phi^{2}$, or equivalently the number of orbits of their face-stabilisers $A_{\phi}$ and $G_{\phi}$ on $\Phi$. Now $A_{\phi} \cong D_{n}$ and $G_{\phi} \cong C_{n}$, and for all $\mathcal{M}$ except the icosahedron their orbits on $\Phi$ are either trivial (fixing $\phi$ and its antipodal face, if it exists), or natural orbits of length $n$. Thus $A_{\phi}$ and $G_{\phi}$ have the same number of orbits on $\Phi$, so $A$ and $G$ have the same rank, as required.

This argument fails for the icosahedron, with $A$ and $G$ having ranks 6 and 8 on $\Phi$, and $A_{\phi}$ having two regular orbits, of length $2 n=6$; in Sect. 7 we will give explicit examples of chiral maps in $E_{p}(\mathcal{M})$ in this case.

\subsection{Maximal submodules and irreducible covers}

If, as we generally assume here, $p$ does not divide $|G|$, then Maschke's Theorem implies that $H_{1}\left(S ; \mathbb{F}_{p}\right)$ is a direct sum of irreducible $G$-submodules.

It follows that each map $\mathcal{N} \in E_{p}(\mathcal{M})$ is the join (minimal common cover) of a finite set of irreducible covering maps $\mathcal{N}_{i} \in E_{p}(\mathcal{M})$.

Here we define any $\mathcal{N}_{i} \in E_{p}(\mathcal{M})$ to be an irreducible covering map if the corresponding submodule $L_{i}$ of $H_{1}\left(S ; \mathbb{F}_{p}\right)$ is maximal, or equivalently the covering group $K_{i} \cong H_{1}\left(S ; \mathbb{F}_{p}\right) / L_{i} \cong \bar{N}_{i}$ is an irreducible $G$-module, so that $\mathcal{N}_{i}$ does not cover any other map in $E_{p}(\mathcal{M})$. The covering group $K$ corresponding to a map $\mathcal{N} \in E_{p}(\mathcal{M})$ is then the direct sum of the covering groups $K_{i}$ corresponding to a set of irreducible covering maps $\mathcal{N}_{i}$. In order to understand the covering maps $\mathcal{N} \in E_{p}(\mathcal{M})$ of a Platonic map $\mathcal{M}$ it is therefore sufficient to understand its irreducible covering maps, so we will pay particular attention to these when dealing with the individual maps $\mathcal{M}$.

\section{The tetrahedron}

Let $\mathcal{M}$ be the tetrahedral map $\{3,3\}$, with $f=4$ faces and $G=$ Aut $^{+} \mathcal{M} \cong A_{4}$. The group $A_{4}$ has four conjugacy classes, consisting of the identity, the three double transpositions, and two mutually inverse classes of four 3-cycles. Its character table is shown in Table 1; here $\chi_{1}$ is the principle character, $\chi_{2}$ and $\chi_{3}$ are the faithful characters of $A_{4} / V_{4} \cong C_{3}$ where $V_{4}$ is the normal Klein four-group, and $\chi_{4}$ is the character of the natural representation $\rho_{n}$ as the rotation group of the tetrahedron, extended from $\mathbb{R}^{3}$ to $\mathbb{C}^{3}$.

Since $G$ acts doubly transitively on $\Phi$, as the natural representation of $A_{4}$, it follows that $\pi=\chi_{1}+\chi_{4}$, so $H_{1}(S ; \mathbb{C})$ is an irreducible $G$-module affording the character $\chi_{4}$. 
Table 1 The character table of $A_{4}$

\begin{tabular}{lllll}
\hline & 1 & $(.).(.)$. & $(\ldots)^{+}$ & $(\ldots)^{-}$ \\
\hline$\chi_{1}$ & 1 & 1 & 1 & 1 \\
$\chi_{2}$ & 1 & 1 & $\omega$ & $\bar{\omega}$ \\
$\chi_{3}$ & 1 & 1 & $\bar{\omega}$ & $\omega$ \\
$\chi_{4}$ & 3 & -1 & 0 & 0 \\
\hline
\end{tabular}

The primes dividing $|G|$ are 2 and 3, so for primes $p>3$ we can regard Table 1 as the character table of $A_{4}$ over $\overline{\mathbb{F}}_{p}$ by suitable reduction $\bmod p$, with $\omega^{2}+\omega+1=0$. In particular, the $G$-module $Q=H_{1}\left(S ; \mathbb{F}_{p}\right)$ is irreducible, affording the character $\chi=\chi_{4}$ of $G$. It follows that in this case $E_{p}(\mathcal{M})$ consists of a single regular map $\mathcal{M}_{0}$ (see Sect. 2.4.1), with $\operatorname{dim}\left(\mathcal{M}_{0}\right)=3$. (If $p=3$ then $Q$ is again irreducible, and the result is the same.) The map $\mathcal{M}_{0}$ has type $\{3 p, 3\}$ and genus $p^{3}-2 p^{2}+1$. Its orientation-preserving automorphism group $\mathrm{Aut}^{+} \mathcal{M}_{0}$ has an elementary abelian normal subgroup $K \cong\left(C_{p}\right)^{3}$; the quotient group, isomorphic to $G$, has an induced action by conjugation on $K$, so that $K$ is a $G$-module over $\mathbb{F}_{p}$, isomorphic to $Q$. The full automorphism group Aut $\mathcal{M}_{0}$ is an extension of $K$ by $A=$ Aut $\mathcal{M} \cong S_{4}$, with $K \cong Q=P / P_{1}$ where we now regard $P$ as the natural permutation module for $S_{4}$; the character of $S_{4}$ on $K$ is that denoted by $\chi_{4}$ in Table 2 (see Sect. 4 ). When $p=3$ or 5 the map $\mathcal{M}_{0}$ has genus 10 or 76 , and appears as the dual of the map R10.1 or R76.2 in Conder's computer-generated list of regular orientable maps [3].

Here it is useful to mention a related result, obtained by similar methods, namely the classification by Conder and Ma [4] of the arc-transitive cubic graphs which arise as regular abelian coverings of the tetrahedral graph $K_{4}$ (among several other cubic graphs).

\section{The cube}

Next we consider coverings of the cube $\mathcal{M}=\{4,3\}$ branched over its face-centres (or, dually, of the octahedron $\{3,4\}$, branched over its vertices). In this case $G=$ $\Delta(3,2,4) \cong S_{4}$, acting on the set $\Phi$ of six faces of $\mathcal{M}$, and the stabilisers of faces are the three subgroups $H \cong C_{4}$ of $G$.

In $S_{4}$ there are five conjugacy classes: the identity, six transpositions, three double transpositions, eight 3-cycles, and six 4-cycles. Hence there are five irreducible characters, shown in Table 2 . In addition to the principal character $\chi_{1}$ and the alternating character $\chi_{2}(g)=\operatorname{sgn}(g), \chi_{3}$ and $\chi_{4}$ are the non-principal irreducible characters obtained from the doubly transitive representations of $S_{4}$ of degrees 3 (via the epimorphism $S_{4} \rightarrow S_{3}$ ) and 4 (the natural action), while $\chi_{5}$ is the character of the natural representation $\rho_{n}$ of $G$ as the rotation group of the cube or octahedron.

The subgroup $H$ consists of the identity element, one double transposition and two 4-cycles. By averaging their values over $H$, one therefore sees that the characters $\chi_{i}$ have multiplicities $1,0,1,0$ and 1 in the permutation character $\pi$ on $P$, that is,

$$
\pi=\chi_{1}+\chi_{3}+\chi_{5}
$$


Table 2 The character table of $S_{4}$

\begin{tabular}{llllll}
\hline & 1 & $(.)$. & $(.).(.)$. & $(\ldots)$ & $(\ldots)$ \\
\hline$\chi_{1}$ & 1 & 1 & 1 & 1 & 1 \\
$\chi_{2}$ & 1 & -1 & 1 & 1 & -1 \\
$\chi_{3}$ & 2 & 0 & 2 & -1 & 0 \\
$\chi_{4}$ & 3 & 1 & -1 & 0 & -1 \\
$\chi_{5}$ & 3 & -1 & -1 & 0 & 1 \\
\hline
\end{tabular}

It follows that the representation of $G$ on $H_{1}(S ; \mathbb{C})$ has character

$$
\chi=\chi_{3}+\chi_{5}
$$

so $H_{1}(S ; \mathbb{C})$ is a direct sum of two irreducible submodules of dimensions 2 and 3.

The primes dividing $|G|$ are 2 and 3, so we can use this decomposition to find the decomposition of $H_{1}\left(S ; \mathbb{F}_{p}\right)$ for any prime $p>3$. In this case $H_{1}\left(S ; \mathbb{F}_{p}\right)$ is also a direct sum of two irreducible submodules of dimensions 2 and 3 , so $E_{p}(\mathcal{M})$ consists of three covering maps $\mathcal{N}$, with $\operatorname{dim}(\mathcal{N})=2,3$ and 5: the first two are irreducible covers of $\mathcal{M}$, and the third is their join. Since the cube has an antipodal symmetry, with the $f=6$ faces permuted in three antipodal pairs, the first two coverings $\mathcal{N}$ are the maps $\mathcal{M}_{a^{\prime}}$ and $\mathcal{M}_{a}$ corresponding to the summands $L=Q_{a^{\prime}}$ and $Q_{a}$ in the $G$-module decomposition $Q=Q_{a} \oplus Q_{a^{\prime}}$ (see Sect. 2.4.3), while the third is $\mathcal{M}_{0}$, corresponding to $L=0$ (see Sect. 2.4.1). These are regular maps of type $\{4 p, 3\}$ and genus $2 p^{c}-3 p^{c-1}+1$ where $c=\operatorname{dim}(\mathcal{N})=2,3$ or 5 , affording the character $\chi_{3}, \chi_{5}$ or $\chi_{3}+\chi_{5}$ respectively; for instance, if $p=5$ or 7 then the maps $\mathcal{M}_{a^{\prime}}$ are the duals of the regular maps R36.3 and R78.1 in [3]. The inclusions $0<Q_{a^{\prime}}$ and $0<Q_{a}$ of $G$-submodules induce regular coverings $\mathcal{M}_{0} \rightarrow \mathcal{M}_{a^{\prime}}$ and $\mathcal{M}_{0} \rightarrow M_{a}$, with covering groups $Q_{a^{\prime}}$ and $Q_{a}$.

Using similar methods, Conder and Ma [4] have classified the arc-transitive graphs which are abelian covers of the 3-cube graph $Q_{3}$, the 1-skeleton of $\mathcal{M}$.

\section{The octahedron}

We now consider coverings of the octahedron $\mathcal{M}=\{3,4\}$ branched over its facecentres (or, dually, of the cube $\{4,3\}$, branched over its vertices). As in the case of the cube we have $G \cong S_{4}$, but now acting on the set $\Phi$ of eight faces of $\mathcal{M}$, so the stabilisers of faces are the four Sylow 3-subgroups $H \cong C_{3}$ of $G$. The conjugacy classes and character table of $G$ are as before, but now $H$ consists of the identity and two 3-cycles. We find that

$$
\pi=\chi_{1}+\chi_{2}+\chi_{4}+\chi_{5}
$$

so

$$
\chi=\chi_{2}+\chi_{4}+\chi_{5}
$$

Thus $H_{1}(S ; \mathbb{C})$ is a direct sum of three irreducible $G$-submodules of dimensions 1,3 and 3. For primes $p>3$ we therefore obtain seven coverings $\mathcal{N} \in E_{p}(\mathcal{M})$ with $c=$ 
$\operatorname{dim}(\mathcal{N})=1,3,3,4,4,6$ and 7, corresponding to the seven proper $G$-submodules $L$ of $Q$; the first three are irreducible covers, and the other four are their joins, two or three at a time.

These are regular maps of type $\{3 p, 4\}$ and genus $g=3 p^{c}-4 p^{c-1}+1$. When $c=1$ we have a regular map $\mathcal{N}$ of genus $3(p-1)$, affording the character $\chi_{2}$ of $G$. The Riemann surfaces underlying these cyclic covers are examples of the well-known surfaces with 8 $(g+3)$ automorphisms discovered by Accola [1] and Maclachlan [18]; for instance, taking $p=5,7,11,13,17,19,23,29$ and 31 we obtain the duals of the regular maps R12.1, R18.1, R30.1, R36.5, R48.1, R54.1, R66.3, R84.1 and R90.1 in [3]. When $c=3$ or $c=4$ we obtain a pair of $p^{c}$-sheeted coverings $\mathcal{N}$ of $\mathcal{M}$; not only are they non-isomorphic, but their automorphism groups are non-isomorphic since the corresponding pairs of normal subgroups $K$ of $\tilde{G}$ are non-isomorphic as $G$-modules, affording characters $\chi_{4}$ and $\chi_{5}$ when $c=3$, or $\chi_{2}+\chi_{4}$ and $\chi_{2}+\chi_{5}$ when $c=4$. The remaining coverings, with $c=6$ and 7 , afford the characters $\chi_{4}+\chi_{5}$ and $\chi_{2}+\chi_{4}+\chi_{5}$.

These $G$-submodules $L \leq Q$, and the corresponding maps $\mathcal{N} \in E_{p}(\mathcal{M})$, all have combinatorial interpretations. The octahedron has an antipodal symmetry, so for each prime $p$ there is a $G$-submodule $Q_{a}$ affording the character $\pi_{a}-\chi_{1}\left(=\chi_{4}\right.$ if $\left.p>3\right)$, giving a regular map $\mathcal{N}=M_{a} \in E_{p}(\mathcal{M})$ with $\operatorname{dim}(\mathcal{N})=4$ and $K \cong Q / Q_{a}$ affording $\chi-\pi_{a}+\chi_{1}\left(=\chi_{2}+\chi_{5}\right.$ if $\left.p>3\right)$. If $p>2$ there is a $G$-invariant complement $Q_{a^{\prime}}$ for $Q_{a}$, giving a regular map $\mathcal{N}=M_{a^{\prime}} \in E_{p}(\mathcal{M})$ with $\operatorname{dim}(\mathcal{N})=3$ and $K \cong Q / Q_{a^{\prime}}$ affording $\pi_{a}-\chi_{1}\left(=\chi_{4}\right.$ if $\left.p>3\right)$.

Since the octahedron is 2 -face-colourable (i.e. its dual, the cube, embeds a bipartite graph), there is a 2-dimensional $G$-submodule $P_{b}>P_{1}$ spanned by the sums of the two monochrome subsets $\Psi \subset \Phi$, and a 5-dimensional $G$-submodule $P_{a}+P_{b}<P^{1}$; these lead, via $G$-submodules $Q_{b}=P_{b} / P_{1}$ and $Q_{a} \oplus Q_{b}$ of $Q$, to coverings $\mathcal{M}_{b}$ and $\mathcal{M}_{a, b}$ in $E_{p}(\mathcal{M})$ of dimensions $c=6$ and 3 respectively. If $p>3$ then since $P_{b}$ affords the character $\chi_{1}+\chi_{2}$ of $G$, these two coverings afford $\chi_{4}+\chi_{5}$ and $\chi_{5}$ respectively. There is also a 7-dimensional $G$-submodule $P_{b^{\prime}}<P$ consisting of the elements $\sum_{\phi \in \Phi} a_{\phi} \phi$ such that $\sum_{\phi \in \Psi} a_{\phi}=\sum_{\phi \in \Psi^{\prime}} a_{\phi}$ where $\Psi$ and $\Psi^{\prime}$ are the monochrome subsets; it contains $P_{1}$, so its image $Q_{b^{\prime}}$ in $Q$ is a 6-dimensional $G$-submodule leadng to a cyclic covering $\mathcal{M}_{b^{\prime}}$ affording $\chi_{2}$. Finally, $Q_{a^{\prime}} \cap Q_{b^{\prime}}$ is a 3-dimensional $G$-submodule of $Q$ (the reduction $\bmod p$ of the natural representation $\rho_{n}$ of $G$ ) giving a 4-dimensional covering $\mathcal{M}_{a^{\prime}, b^{\prime}}$ affording $\chi_{2}+\chi_{4}$. Together with $\mathcal{M}_{0}$ this accounts for all seven maps in $E_{p}(\mathcal{M})$ when $p>3$.

The direct sum decomposition $Q=Q_{b} \oplus Q_{a} \oplus\left(Q_{a^{\prime}} \cap Q_{b^{\prime}}\right)$, with direct factors affording the irreducible representations $\rho_{2}, \rho_{4}$ and $\rho_{5}$ corresponding to the characters $\chi_{2}, \chi_{4}$ and $\chi_{5}$, is also valid when $p=3$. (The character $\chi_{3}$, which reduces mod 3 to $\chi_{1}+\chi_{2}$, is not a summand of $\chi$; the remaining four characters in Table 2 correspond to irreducible characters over $\mathbb{F}_{3}$.) Thus $E_{3}(\mathcal{M})$ consists of the seven regular maps described above.

The dimensions $c=1,3,4,6$ and 7 have also been obtained by Kwak and Oh [17] in their classification of arc-transitive elementary abelian coverings of the octahedral graph: they find $p+4$ such covering for each prime $p>2$ (and 14 when $p=2$ ). Of course, these include the 1-skeletons of the maps in $E_{p}(\mathcal{M})$. 
Table 3 The character table of $A_{5}$

\begin{tabular}{llllll}
\hline & 1 & $(.).(.)$. & $(\ldots)$ & $(\ldots . .)^{+}$ & $(\ldots . .)^{-}$ \\
\hline$\chi_{1}$ & 1 & 1 & 1 & 1 & 1 \\
$\chi_{2}$ & 3 & -1 & 0 & $\lambda$ & $\mu$ \\
$\chi_{3}$ & 3 & -1 & 0 & $\mu$ & $\lambda$ \\
$\chi_{4}$ & 4 & 0 & 1 & -1 & -1 \\
$\chi_{5}$ & 5 & 1 & -1 & 0 & 0 \\
\hline
\end{tabular}

\section{The dodecahedron}

We now consider coverings of the dodecahedron $\mathcal{M}=\{5,3\}$ branched over its facecentres (or, dually, of the icosahedron $\{3,5\}$, branched over its vertices). In this case $G=\Delta(3,2,5) \cong A_{5}$, acting on the set $\Phi$ of twelve faces of $\mathcal{M}$, so the stabilisers of faces are the Sylow 5-subgroups $H \cong C_{5}$ of $G$. The maps $\mathcal{N} \in E_{p}(\mathcal{M})$ have type $\{5 p, 3\}$ and genus $5 p^{c}-6 p^{c-1}+1$ where $c=\operatorname{dim}(\mathcal{N})$.

There are five conjugacy classes in $A_{5}$, the identity, fifteen double transpositions, twenty 3-cycles, and two classes of twelve 5-cycles, so there are five irreducible characters. The character table is as shown in Table 3 , with $\lambda, \mu=(1 \pm \sqrt{5}) / 2$.

In addition to the principal character $\chi_{1}$, there are algebraically conjugate characters $\chi_{2}$ and $\chi_{3}$ obtained from the natural representation $\rho_{n}$ of $G$ as a rotation group, while the irreducible characters $\chi_{4}$ and $\chi_{5}$ are the non-principal summands of the permutation characters corresponding to the doubly transitive natural permutations representations of $G$ as $A_{5}$ and as $\operatorname{PSL}_{2}(5)$.

The subgroup $H$ consists of the identity element, and two elements each from the two conjugacy classes of 5-cycles. By averaging their values over $H$, one sees that the characters $\chi_{i}$ have multiplicities $1,1,1,0$ and 1 in the permutation character $\pi$ on $P$, that is,

$$
\pi=\chi_{1}+\chi_{2}+\chi_{3}+\chi_{5}
$$

Thus

$$
\chi=\chi_{2}+\chi_{3}+\chi_{5}
$$

so $H_{1}(S ; \mathbb{C})$ is a direct sum of three irreducible submodules of dimensions 3,3 and 5 .

The primes dividing $|G|$ are 2, 3 and 5, so we can use this decomposition to find the decomposition of $H_{1}\left(S ; \mathbb{F}_{p}\right)$ for any prime $p>5$. If $p \equiv \pm 1(\bmod 5)$, so that 5 has a square root in $\mathbb{F}_{p}$, then $H_{1}\left(S ; \mathbb{F}_{p}\right)$ is also a direct sum of three irreducible submodules of dimensions 3, 3 and 5; in this case we obtain seven covering maps $\mathcal{N} \in E_{p}(\mathcal{M})$, of dimensions $c=3,3,5,6,8,8$ and 11 , with the first three irreducible. If $p \equiv \pm 2$ $(\bmod 5)$, on the other hand, 5 has no square root in $\mathbb{F}_{p}$; in this case $H_{1}\left(S ; \mathbb{F}_{p}\right)$ is a direct sum of irreducible submodules of dimensions 5 and 6 , and there are three covering maps $\mathcal{N} \in E_{p}(\mathcal{M})$, with $c=5,6$ and 11; the first two are irreducible. In either case, the maps in $E_{p}(\mathcal{M})$ are all regular; those with $c=5$ and 6 are $\mathcal{M}_{a^{\prime}}$ 
and $\mathcal{M}_{a}$, arising from the antipodal symmetry of the dodecahedron, while $\mathcal{M}_{0}$ has $c=11$; these coverings afford the characters $\chi_{5}, \chi_{2}+\chi_{3}$ and $\chi$ respectively. When $p \equiv \pm 1(\bmod 5)$ there are two 3-dimensional submodules $Q_{n}$ and $Q_{n *}$, obtained from the natural representation $\rho_{n}$ and its algebraic conjugate $\rho_{n *}$; these lead to maps $\mathcal{M}_{n}$ and $\mathcal{M}_{n *}$ in $E_{p}(\mathcal{M})$ with $c=8$, affording $\chi_{2}+\chi_{5}$ and $\chi_{3}+\chi_{5}$. Finally, in this case there are also two 8-dimensional submodules $Q_{a} \oplus Q_{n}$ and $Q_{a} \oplus Q_{n *}$, leading to irreducible 3-dimensional coverings $\mathcal{M}_{a, n}$ and $\mathcal{M}_{a, n *}$ affording $\chi_{3}$ and $\chi_{2}$.

The direct sum decomposition $Q=Q_{a} \oplus Q_{a^{\prime}}$, with direct factors affording the characters $\chi_{5}$ and $\chi_{2}+\chi_{3}$, is in fact also valid when $p=3$ or 5 . Over $\mathbb{F}_{3}$, the character $\chi_{5}$ splits as $\chi_{1}+\chi_{4}$, while $\chi_{2}+\chi_{3}$ is irreducible (but splits into two algebraically conjugate characters over $\mathbb{F}_{9}$ ).

\section{The icosahedron}

We now consider coverings of the icosahedron $\mathcal{M}=\{3,5\}$ branched over its facecentres (or, dually, of the dodecahedron $\{5,3\}$, branched over its vertices). The maps $\mathcal{N} \in E_{p}(\mathcal{M})$ have type $\{3 p, 5\}$ and genus $9 p^{c}-10 p^{c-1}+1$, where $c=\operatorname{dim}(\mathcal{N})$.

As in the case of the dodecahedron we have $G \cong A_{5}$, but now acting on the set $\Phi$ of twenty faces of $\mathcal{M}$, so the stabilisers of faces are the ten Sylow 3-subgroups $H \cong C_{3}$ of $G$. The conjugacy classes and character table of $G$ are as before, but now $H$ consists of the identity and two 3 -cycles. In this case we find that

$$
\pi=\chi_{1}+\chi_{2}+\chi_{3}+2 \chi_{4}+\chi_{5}
$$

so

$$
\chi=\chi_{2}+\chi_{3}+2 \chi_{4}+\chi_{5}
$$

Thus $H_{1}(S ; \mathbb{C})$ is a direct sum of irreducible submodules of dimensions $3,3,4$ (with multiplicity 2) and 5. In contrast with the previous examples, the existence of a summand with multiplicity greater than 1 implies that the direct sum decompositions of $\mathbb{C} \Phi$ and of $H_{1}(S ; \mathbb{C})$ are not unique: in each case the 8-dimensional submodule affording the character $2 \chi_{4}$ contains infinitely many mutually isomorphic 4-dimensional irreducible submodules affording $\chi_{4}$ (see Sect. 2.5).

As in the case of the dodecahedron, we can use this to obtain the decomposition of $H_{1}\left(S ; \mathbb{F}_{p}\right)$ for primes $p>5$, but first we need to consider this lack of uniqueness of 4-dimensional submodules.

There is a chiral pair of $G$-invariant equivalence relations $\sim_{b}$ and $\sim_{c}$ on $\Phi$, each with five classes of four faces, corresponding to the inclusion of $H$ in two subgroups of $G$ isomorphic to $A_{4}$. (These are the well-known five-colourings of the faces of $\mathcal{M}$, often used to demonstrate the isomorphism of $G$ with $A_{5}$.) Their equivalence classes $\Psi$ provide basis elements $\underline{\Psi}$ for a pair of 5-dimensional submodules $P_{b}, P_{c}>P_{1}$ of $P$, and hence a pair of 4-dimensional submodules $Q_{b}$ and $Q_{c}$ of $Q$. There is an isomorphism $Q_{b} \rightarrow Q_{c}, q \mapsto q^{\prime}$, induced by the antipodal automorphism $i$ of $\mathcal{M}$, and these submodules each afford the character $\chi_{4}$ which has multiplicity 2 in the 
decomposition of $\chi$. They generate an 8-dimensional submodule $Q_{b, c}=Q_{b} \oplus Q_{c}$ which contains $p-1$ other submodules isomorphic to them, each of the form $Q(\lambda)=$ $\left\{q+\lambda q^{\prime} \mid q \in Q_{b}\right\}$ for some $\lambda \in \mathbb{F}_{p}^{*}$ (see Sect. 2.5). Now $i$ transposes $\sim_{b}$ and $\sim_{c}$, and hence also transposes $Q(0):=Q_{b}$ and $Q(\infty):=Q_{c}$, so it permutes these $p+1$ submodules $Q(\lambda)\left(\lambda \in \mathbb{P}^{1}(p)=\mathbb{F}_{p} \cup\{\infty\}\right)$, leaving $Q(1)$ and $Q(-1)$ (if $p>2$ ) invariant and otherwise transposing pairs $Q\left(\lambda^{ \pm 1}\right)$. Since $A=G \times\langle i\rangle$, the $G$ submodules $Q( \pm 1)$ are actually $A$-submodules, affording the irreducible characters $\chi_{4} \otimes \varphi_{ \pm 1}$ of $A$, where $\varphi_{1}$ and $\varphi_{-1}$ are the principal and non-principal characters of $\langle i\rangle \cong C_{2}$.

If $p \equiv \pm 1(\bmod 5)$ then $H_{1}\left(S ; \mathbb{F}_{p}\right)$ is a direct sum of irreducible submodules of the same dimensions and multiplicities as those for $H_{1}(S ; \mathbb{C})$. The two irreducible submodules $Q_{n}$ and $Q_{n *}$ of dimension 3 are obtained from the natural representation of $G$, those of dimension 4 are the submodules $Q(\lambda)<Q_{b, c}$ defined above, and there is also one irreducible submodule $Q_{d}$ of dimension 5. (This is the image in $Q$ of the 6-dimensional submodule of $P$ spanned by the elements $\underline{\Psi}$, where $\Psi$ consists of the ten faces of $\mathcal{M}$ incident with an antipodal pair of vertices; these six elements are permuted by $G$ as it permutes the diagonals of $\mathcal{M}$, or equivalently as $\operatorname{PSL}_{2}(5)$ permutes $\mathbb{P}^{1}(5)$.) There are eight submodules intersecting $Q_{b, c}$ trivially, namely the various sums of $Q_{n}, Q_{n *}$ and $Q_{d}$, with codimensions $c=8,11,11,13,14,16,16$ and 19; by taking the direct sum of one of these with a submodule $Q_{b, c}$ or $Q(\lambda)$ we obtain eight more submodules with codimensions $0,3,3,5,6,8,8$ and 11 , or $8(p+1)$ with codimensions $4,7,7,9,10,12,12$ and 15 . Excluding the submodule $Q$ of codimension 0 , this gives a total of $8 p+23$ proper submodules, and hence the same number of covering maps $\mathcal{N} \in E_{p}(\mathcal{M})$, with $\operatorname{dim}(\mathcal{N})=3,4, \ldots, 16$ and 19. The irreducible coverings are the two, $p+1$ and one of dimensions 3, 4 and 5. Of the maps in $E_{p}(\mathcal{N}), 8(p-1)$ are chiral, namely those corresponding to submodules containing a single submodule $Q(\lambda)$, with $\lambda \neq \pm 1$, while the remaining $8+16+7=31$ are regular.

If $3<p \equiv \pm 2(\bmod 5)$ then $H_{1}\left(S ; \mathbb{F}_{p}\right)$ can be decomposed as the direct sum of four irreducible submodules: two are isomorphic 4-dimensional submodules $Q(\lambda)<Q_{b, c}$, affording $\chi_{4}$, and the other two are $Q_{d}$ of dimension 5, affording $\chi_{5}$, and $Q_{e}:=Q_{n} \oplus Q_{n *}$ of dimension 6, affording $\chi_{2}+\chi_{3}$. There are four submodules intersecting $Q_{b, c}$ trivially, namely $Q_{d} \oplus Q_{e}, Q_{e}, Q_{d}$ and 0 with codimensions 8, 13, 14 and 19; by taking the direct sums of these with a submodule $Q_{b, c}$ or $Q(\lambda)$ we obtain four more submodules with codimensions $0,5,6$ and 11 , or $4(p+1)$ with codimensions $4,9,10$ and 15 . Excluding $Q$ as before, we obtain $4 p+11$ proper submodules, and the same number of covering maps $\mathcal{N} \in E_{p}(\mathcal{M})$, with $\operatorname{dim}(\mathcal{N})=4,5,6,8,9,10,11,13,14,15$ and 19 . There are $p+1$ irreducible coverings of dimension 4 , and one each of dimensions 5 and 6 . There are $4(p-1)$ chiral maps in $E_{p}(\mathcal{M})$, corresponding to submodules containing a single $Q(\lambda)$, with $\lambda \neq \pm 1$, while the other 15 maps are regular.

In either case, the covering maps $\mathcal{N}=\mathcal{M}_{a}$ and $\mathcal{M}_{a^{\prime}}$ obtained from the antipodal symmetry of $\mathcal{M}$ arise as above from the submodules $Q_{a}=Q_{d} \oplus Q(1)$ and $Q_{a^{\prime}}=$ $Q_{e} \oplus Q(-1)$. 


\section{The dihedron}

If $\mathcal{M}$ is the dihedron $\{n, 2\}$, then

$$
G=\Delta(2,2, n)=\left\langle x, y, z \mid x^{2}=y^{2}=z^{n}=x y z=1\right\rangle .
$$

This can be identified with the dihedral group

$$
D_{n}=\left\langle a, b \mid a^{n}=b^{2}=1, a^{b}=a^{-1}\right\rangle,
$$

where $x=b$ and $y=a b$ are rotations through $\pi$, fixing a vertex and edge-centre respectively and transposing the two faces, and $z=a$ is a rotation through $2 \pi / n$, preserving the faces, so that $H=\langle a\rangle \cong C_{n}$. Then $H_{1}(S ; \mathbb{Z})$ is an infinite cyclic group, with $a$ acting on it as the identity and $b$ inverting every element. Thus

$$
\pi=\chi_{1}+\chi_{2}
$$

and hence

$$
\chi=\chi_{2}
$$

where $\chi_{1}$ is the principal character of $G$ and $\chi_{2}(g)=(-1)^{k}$ for each $g=a^{j} b^{k} \in G$. It follows that for each integer $e \geq 1$ there is a single regular covering $\mathcal{N}$ of exponent $e$, with a cyclic covering group, corresponding to the unique subgroup of index $e$ in $H_{1}(S ; \mathbb{Z})$. This map is the dihedron $\{e n, 2\}$, a regular map of genus 0 with $G \cong D_{e n}$ and $A=G \times\langle c\rangle \cong D_{e n} \times C_{2}$, where $c$ is the reflection fixing all the vertices and edges of $\mathcal{N}$ and transposing its two faces.

\section{The hosohedron}

If $\mathcal{M}$ is the hosohedron $\{2, n\}$, then for each prime $p$ the maps $\mathcal{N} \in E_{p}(\mathcal{M})$ are all regular, of type $\{2 p, n\}$ and genus

$$
1+\frac{p^{c-1}}{2}(n(p-1)-2 p),
$$

where $c=\operatorname{dim}(\mathcal{N})$. As in the case of the dihedron, $G$ is isomorphic to $D_{n}$, but now acting naturally with degree $n$ on $\Phi$, so that the subgroup stabilising a face is $H=\langle b\rangle \cong C_{2}$. This makes the analysis of the coverings more complicated than before.

\subsection{Conjugacy classes and characters of $D_{n}$}

First suppose that $n$ is odd. Then apart from the conjugacy class $\{1\}$, there are $(n-1) / 2$ conjugacy classes $\left\{a^{ \pm j}\right\}$ for $j=1,2, \ldots,(n-1) / 2$, and a single conjugacy class $\left\{a^{j} b \mid j=0,1, \ldots, n-1\right\}$ consisting of the involutions in $G$. 
Table 4 The character table of $D_{n}$ for odd $n$

\begin{tabular}{llll}
\hline & 1 & $a^{ \pm j}$ & $a^{j} b$ \\
\hline$\chi_{1}$ & 1 & 1 & 1 \\
$\chi_{2}$ & 1 & 1 & -1 \\
$\xi_{k}$ & 2 & $\alpha_{j k}$ & 0 \\
\hline
\end{tabular}

Table 5 The character table of $D_{n}$ for even $n$

\begin{tabular}{llll}
\hline & 1 & $a^{ \pm j}$ & $a^{j} b$ \\
\hline$\chi_{1}$ & 1 & 1 & 1 \\
$\chi_{2}$ & 1 & 1 & -1 \\
$\chi_{3}$ & 1 & $(-1)^{j}$ & $(-1)^{j}$ \\
$\chi_{4}$ & 1 & $(-1)^{j}$ & $(-1)^{j+1}$ \\
$\xi_{k}$ & 2 & $\alpha_{j k}$ & 0 \\
\hline
\end{tabular}

In addition to the characters $\chi_{1}$ and $\chi_{2}$ of degree 1 defined in the preceding section, $G$ has $(n-1) / 2$ irreducible characters $\xi_{k}$ of degree 2 , for $k=1,2, \ldots,(n-1) / 2$, where $\xi_{k}\left(a^{ \pm j}\right)=\alpha_{j k}:=\zeta_{n}^{j k}+\zeta_{n}^{-j k}$ with $\zeta_{n}=\exp (2 \pi i / n)$. The character table of $G$ is as in Table 4, where the column headed $a^{ \pm j}$ and the row labelled $\xi_{k}$ represent $(n-1) / 2$ conjugacy classes and characters respectively, as $j$ and $k$ each range over $\{1,2, \ldots,(n-1) / 2\}$. For each $k$, the kernel of the representation $\rho_{k}$ corresponding to $\xi_{k}$ is the unique subgroup $\left\langle a^{m}\right\rangle$ of $\operatorname{order} \operatorname{gcd}(k, n)$ in $G_{0}:=\langle a\rangle$, where $m=$ $n / \operatorname{gcd}(k, n) \geq 3$, so $\rho_{k}$ can be regarded as a faithful representation of $G /\left\langle a^{m}\right\rangle \cong D_{m}$.

By computing the inner product of each irreducible character with $\pi$ we find that

$$
\pi=\chi_{1}+\sum_{k=1}^{(n-1) / 2} \xi_{k}
$$

and hence

$$
\chi=\sum_{k=1}^{(n-1) / 2} \xi_{k}
$$

If $n$ is even the conjugacy classes of $G$ are $\{1\},\left\{a^{n / 2}\right\},\left\{a^{ \pm j}\right\}$ for $j=1,2, \ldots,(n-$ 2) $/ 2,\left\{a^{j} b \mid j\right.$ is even $\}$ and $\left\{a^{j} b \mid j\right.$ is odd $\}$. In addition to $\chi_{1}$ and $\chi_{2}$, defined as above for $n$ odd, there are two more characters $\chi_{3}$ and $\chi_{4}$ of degree 1 , together with irreducible characters $\xi_{k}$ of degree 2 for $k=1,2, \ldots,(n-2) / 2$. The character table is as in Table 5, with the columns headed $a^{ \pm j}$ and $a^{j} b$ representing $1+(n-2) / 2=n / 2$ and 2 conjugacy classes respectively, and the row labelled $\xi_{k}$ representing $(n-2) / 2$ characters. We can take $H=\langle b\rangle$, so that

$$
\pi=\chi_{1}+\chi_{3}+\sum_{k=1}^{(n-2) / 2} \xi_{k}
$$


and hence

$$
\chi=\chi_{3}+\sum_{k=1}^{(n-2) / 2} \xi_{k} .
$$

\subsection{Submodule structure}

Instead of considering the reduction mod $p$ of the characters appearing in $\pi$, as in the preceding cases, it is more convenient to use the fact that the group $G=D_{n}$ has a subgroup $G_{0}=\langle a\rangle$ acting regularly on $\Phi$. This allows a more explicit description of the $G$-submodules of $P$ and $Q$, and hence of the maps $\mathcal{N} \in E_{p}(\mathcal{M})$. We will assume that $p$ is coprime to $n$. As in Sect. 2, certain key observations are italicised in the preliminary explanation of this method.

In order to find the $G$-module decompositions of $P=\mathbb{F}_{p} \Phi$, and hence of $H_{1}\left(S ; \mathbb{F}_{p}\right) \cong Q=P / P_{1}$, we first consider them as $G_{0}$-modules. Since $G_{0}$ acts regularly on $\Phi$, one can identify $P$ with the group algebra $\mathbb{F}_{p} G_{0}$ of $G_{0}$ over $\mathbb{F}_{p}$, or equivalently with the polynomial algebra $\mathbb{F}_{p}[x] /\left(x^{n}-1\right)$, so that the elements $a^{j} \in G_{0}$ correspond to the images of the powers $x^{j}$ of $x$.

The $G_{0}$-submodules are then the ideals of $\mathbb{F}_{p}[x] /\left(x^{n}-1\right)$, each generated by the image of the ideal in $\mathbb{F}_{p}[x]$ generated by a polynomial dividing $x^{n}-1$.

To find these polynomials we need to determine the irreducible factors of $x^{n}-1$ in $\mathbb{F}_{p}[x]$. In $\mathbb{Z}[x]$ we have a factorisation

$$
x^{n}-1=\prod_{m \mid n} \Phi_{m}(x),
$$

where $\Phi_{m}(x)$ is the $m$-th cyclotomic polynomial, the minimal polynomial of the primitive $m$-th roots of 1 in $\mathbb{C}$. In fact, equation (1), together with the equation $\Phi_{1}(x)=x-1$, can be regarded as a recursive definition of $\Phi_{n}(x)$ in terms of the polynomials $\Phi_{m}(x)$ for the proper divisors $m$ of $n$.

Each cyclotomic polynomial is irreducible in $\mathbb{Z}[x]$ (in fact, in $\mathbb{Q}[x]$ ), but in almost all cases it factorises when reduced $\bmod p$, that is, when regarded as a polynomial in $\mathbb{F}_{p}[x]$. We are assuming that $n$ is coprime to $p$, so the set $\Omega=\Omega_{n}$ of $n$-th roots of 1 in the algebraic closure $\overline{\mathbb{F}}_{p}$ of $\mathbb{F}_{p}$ contains $n$ elements. This set is partitioned into disjoint subsets $\Pi_{m}(m \mid n)$ consisting of the $\varphi(m)$ primitive $m$-th roots of 1 in $\overline{\mathbb{F}}_{p}$. For each divisor $m$ of $n$, let $e=e_{m}$ denote the multiplicative order of $p$ as a unit $\bmod m$, so that $m$ divides $p^{e}-1$ but not $p^{f}-1$ for any $f<e$. Then each $\omega \in \Pi_{m}$ generates the subfield $\mathbb{F}_{p^{e}}$ of $\overline{\mathbb{F}}_{p}$. The restriction to $\mathbb{F}_{p^{e}}$ of the Frobenius automorphism $\phi: t \mapsto t^{p}$ of $\overline{\mathbb{F}}_{p}$ generates the Galois group $C=\mathrm{Gal} \mathbb{F}_{p^{e}} \cong C_{e}$ of this subfield, which partitions $\Pi_{m}$ into $\varphi(m) / e$ orbits

$$
\Gamma=\left\{\omega, \omega^{p}, \ldots, \omega^{p^{e-1}}\right\}
$$


of length $e$. Each such orbit $\Gamma$ is the set of roots $\omega^{p^{i}}$ of an irreducible monic factor $f^{\Gamma}(x)$ of degree $e$ of $\Phi_{m}(x)$ in $\mathbb{F}_{p}[x]$.

As $\Gamma$ ranges over the orbits of the Galois group $C$ on $\Omega$, these polynomials $f^{\Gamma}(x)$ give all the irreducible factors of $x^{n}-1$, and the ideals $P^{\Gamma}$ they generate give all the maximal $G_{0}$-submodules of $P$.

By taking the ideals generated by all products of these irreducible factors we obtain all the $G_{0}$-submodules of $P$ as intersections of these maximal submodules. In particular, $P$ is the direct sum of the irreducible $G_{0}$-submodules $P_{\Gamma} \cong P / P^{\Gamma}$ generated by the complementary factors $f_{\Gamma}(x)=\left(x^{n}-1\right) / f^{\Gamma}(x)$ of $x^{n}-1$, with the generator $a$ of $G_{0}$ having minimal polynomial $f^{\Gamma}(x)$ on $P_{\Gamma}$. For instance, the orbit $\Gamma=\Pi_{1}=\{1\}$ corresponds to the submodule $P_{1}$ fixed by $G_{0}$; this is generated by

$$
\frac{x^{n}-1}{x-1}=x^{n-1}+\cdots+x+1,
$$

the product of all the irreducible factors except $\Phi_{1}(x)=x-1$. More generally, if $m$ divides $\operatorname{gcd}(n, p-1)$ then $\Pi_{m}$ consists of $\varphi(m)$ singleton orbits $\Gamma=\{\omega\}$, each giving a linear factor $f^{\Gamma}(x)=x-\omega$ of $\Phi_{n}(x)$ and corresponding to a 1-dimensional direct summand $P_{\Gamma}$ of $P$ on which $a$ has eigenvalue $\omega$.

To understand the $G$-module structures of $P$ and $Q$, we need to consider the action of $b$, acting by conjugation on $G_{0}$ as inversion $\iota: a^{j} \mapsto a^{-j}$.

Given any orbit $\Gamma \subseteq \Pi_{m}$ of the Galois group $C=\langle\phi\rangle$ on $\Omega$, the inverses of its elements also form such an orbit $\Gamma^{*}$, so that $\Delta=\Gamma \cup \Gamma^{*}$ is an orbit of $B:=\langle\phi, \iota\rangle$. The orbit $\Gamma$ containing $\omega$ coincides with $\Gamma^{*}$ if and only if

$$
\omega^{p^{f}}=\omega^{-1} \text {, that is, } p^{f} \equiv-1 \quad(\bmod m),
$$

for some integer $f$. (This is always true if $m=1$ or 2 , but otherwise it implies that $e$ is even and $f \equiv e / 2(\bmod e)$.) This condition depends only on $p$ and $m$, so for a given $m$, either all of the orbits $\Gamma \subseteq \Pi_{m}$ of $C$ are self-inverse, each forming an orbit $\Delta$ of $B$, or none of them are, so that each orbit $\Delta$ of $B$ is a union of two mutually inverse orbits $\Gamma$ and $\Gamma^{*}$ of $C$. In the first case, the $G_{0}$-submodules $P^{\Gamma}$ and $P_{\Gamma}$ of $P$ are also $G$-modules, with $P_{\Gamma} \cong P / P^{\Gamma}$ irreducible and $e$-dimensional, whereas in the second case $P_{\Gamma} \oplus P_{\Gamma^{*}}$ is an irreducible $2 e$-dimensional $G$-submodule, isomorphic to $P /\left(P^{\Gamma} \cap P^{\Gamma^{*}}\right)$, with $b$ transposing the two direct factors. For instance, any 1dimensional $G_{0}$-submodules $P_{\Gamma}$ are of the second type, apart those with $m=1$ or 2 . In either case, we will denote this irreducible submodule by $R_{\Delta}$, and we let $d=e$ or $2 e$ denote its dimension. The kernel of the representation of $G$ on $R_{\Delta}$ is the normal subgroup $\left\langle a^{m}\right\rangle \cong C_{n / m}$, so that $G$ acts on $R_{\Delta}$ as $G /\left\langle a^{m}\right\rangle \cong D_{m}$.

These $G$-submodules $R_{\Delta}$ are the irreducible summands in the direct sum decomposition of the G-module $P=P_{1} \oplus P^{1}$; deleting the 1-dimensional submodule $P_{1}$ gives the corresponding decomposition of $Q=P / P_{1} \cong P^{1}$.

We therefore know all the proper $G$-submodules $L$ of $Q$ and hence all the maps $\mathcal{N}$ in $E_{p}(\mathcal{M})$. The possible values of $c=\operatorname{dim}(\mathcal{N})$ range from 1 (corresponding to $L=P^{\Delta} / P_{1}$ where $n$ is even and $\Delta=\Pi_{2}=\{-1\}$ ) to $n-1$ (for $\mathcal{N}=\mathcal{M}_{0}$, corresponding to $L=0$ ). The number $v$ of irreducible summands $R_{\Delta}$ in the direct 
sum decomposition of the $G$-module $Q$ is equal to the number of orbits $\Delta \neq\{1\}$ of $B$ on $\Omega$. Since these summands are mutually non-isomorphic, the total number of $G$-submodules of $Q$ is $2^{v}$; thus $Q$ has $2^{v}-1$ proper $G$-submodules and hence $\left|E_{p}(\mathcal{M})\right|=2^{v}-1$.

This completes the proof of Theorem 1 . The rest of this section consists of examples illustrating the methods applied to the hosohedron, for various $n$.

\subsection{Examples}

To illustrate the preceding arguments, let $n=95$ and $p=7$, so $G \cong D_{95}$. To decompose $P$ and hence $Q$ as direct sums, we consider the divisors $m=1,5,19$ and 95 of $n$ in turn.

The divisor $m=1$ leads to a single orbit $\Delta=\Pi_{1}=\{1\}$ of $B$, with the corresponding 1 -dimensional direct summand $R_{\Delta}=P_{1}$ of $P$, generated by $\left(x^{95}-1\right) /(x-1)$ and affording the principal representation of $G$.

Next we consider $m=5$. The prime $p=7$ has order $e=4$ in $\mathbb{Z}_{5}^{*}$; since $\varphi(5)=4$, the Galois group $C=\operatorname{Gal} \mathbb{F}_{7^{4}} \cong C_{4}$ has $\varphi(5) / e=1$ orbit $\Gamma$ on the set $\Pi_{5} \subset \mathbb{F}_{7^{4}} \subset \overline{\mathbb{F}}_{7}$ of primitive 5 th roots of 1 . The cyclotomic polynomial $\Phi_{5}(x)=f^{\Gamma}(x)=x^{4}+x^{3}+$ $x^{2}+x+1$ is therefore irreducible in $\mathbb{F}_{7}[x]$, giving a 4-dimensional irreducible $G_{0^{-}}$ submodule $P_{\Gamma}=\left(x^{95}-1\right) /\left(f^{\Gamma}(x)\right)$ of $P$. Since $-1 \in\langle 7\rangle$ in $\mathbb{Z}_{5}^{*}$, this is also a 4-dimensional irreducible $G$-submodule $R_{\Delta}$, with $G$ acting on it as $D_{5}$. When we factor out $P_{1}$, this appears as a direct summand of $Q=H_{1}\left(S ; \mathbb{F}_{7}\right)$.

Now let $m=19$. Since 7 has order $e=3$ in $\mathbb{Z}_{19}^{*}$, and there are $\varphi(19)=18$ primitive 19th roots of 1 in $\mathbb{F}_{7^{3}}$, forming six orbits $\Gamma$ under the Galois group $C \cong C_{3}$ of this field, we obtain six 3-dimensional irreducible $G_{0}$-submodules $P_{\Gamma}$ as summands of $P$, corresponding to six irreducible cubic factors $f^{\Gamma}(x)$ of $\Phi_{19}(x)$ in $\mathbb{F}_{7}[x]$. Since $-1 \notin\langle 7\rangle$ in $\mathbb{Z}_{19}^{*}$, these six orbits form three mutually inverse pairs $\Gamma$ and $\Gamma^{*}$, so they merge to give three orbits $\Delta=\Gamma \cup \Gamma^{*}$ of $B$. These correspond to three 6-dimensional irreducible $G$-submodules $R_{\Delta}=P_{\Gamma} \oplus P_{\Gamma^{*}}$ of $P$, and hence also of $Q$, with $G$ acting on each as $D_{19}$.

Finally let $m=95$. Since $\mathbb{Z}_{95} \cong \mathbb{Z}_{5} \oplus \mathbb{Z}_{19}$ we see that 7 has order $e=\operatorname{lcm}\{4,3\}=$ 12 in $\mathbb{Z}_{95}^{*}$, so $\Phi_{95}(x)$ is a product of $\varphi(95) / 12=6$ irreducible polynomials $f^{\Gamma}(x)$ of degree 12 in $\mathbb{F}_{7}[x]$, giving six 12-dimensional irreducible $G_{0}$-submodules $P_{\Gamma}$ as summands of $P$. Since $-1 \notin\langle 7\rangle$ in $\mathbb{Z}_{19}^{*}$ we have $-1 \notin\langle 7\rangle$ in $\mathbb{Z}_{95}^{*}$, so these give three 24-dimensional irreducible $G$-submodules $R_{\Delta}=P_{\Gamma} \oplus P_{\Gamma^{*}}$ of $P$, and hence of $Q$, with $G$ acting faithfully on each.

Adding up the dimensions of these irreducible submodules $R_{\Delta}$, we have

$$
1+4+3 \times 6+3 \times 24=95=\operatorname{dim} P
$$

so they give the complete direct sum decomposition $\oplus_{\Delta} R_{\Delta}$ of $P$ as a $G$-module; deleting the 1-dimensional submodule $P_{1}$ gives the corresponding decomposition of $Q$. Since $Q$ has $v=7$ mutually non-isomorphic irreducible summands, we obtain $2^{7}-1=127$ proper $G$-submodules $L<Q$. Thus $E_{7}(\mathcal{M})$ consists of 127 regular 
maps $\mathcal{N}$, with $\operatorname{dim}(\mathcal{N})=4$ (one map), 6 (three maps), and so on, up to 94 (one map, $\left.\mathcal{M}_{0}\right)$.

Examples with even $n$ can be treated similarly, except that now there is always at least one 1-dimensional summand $R_{\Delta}$, corresponding to the orbit $\Delta=\Gamma=\Pi_{2}=$ $\{-1\}$ of $B$, with $f^{\Gamma}(x)=\Phi_{2}(x)=x+1$. This corresponds to a cyclic covering $\mathcal{N}$ on which $a$ has eigenvalue -1 (see [13, Sect. 9(d)]).

For instance, if $n=4$ then $E_{p}(\mathcal{M})$ consists of regular maps of type $\{2 p, 4\}$ and genus $g=1+p^{e-1}(p-2)$. If $p \neq 2$ then $B$ has orbits $\Delta=\{1\},\{-1\}$ and $\left\{\zeta_{4}^{ \pm 1}\right\}$ on $\Omega_{4}$, the last two providing irreducible $G$-submodules of $Q$ of dimensions 1 and 2, affording the characters $\chi_{3}$ and $\xi_{1}$ of $G$. Thus $E_{p}(\mathcal{M})$ consists of three maps $\mathcal{N}$ with $c=\operatorname{dim}(\mathcal{N})=1,2$ and 3 , of genus $g=p-1,(p-1)^{2}$ and $(p-1)\left(p^{2}-p-1\right)$, affording the characters $\chi_{3}, \xi_{1}$ and $\chi_{3}+\xi_{1}$. The maps with $c=1$ lie on the AccolaMaclachlan surfaces with $8(g+1)$ automorphisms [1,18]; for $p=3,5,7$ and 11 they are the duals of the regular maps R2.2, R4.4, R6.4 and R10.11 in [3]. For the same primes, the maps with $c=2$ (the maps $\{2 p, 4\}_{4}$ in [6, Sect. 8.6 and Table 8]) are the duals of R4.3, R16.3, R36.4 and R100.5 in [3].

\subsection{Prime $n$}

If $n$ is prime then the only divisors $m$ of $n$ are $m=1$, giving rise to the $G$-submodule $P_{1} \leq P$, and $m=n$. If $p \neq n$ then $\Phi_{n}(x)$ factorises in $\mathbb{F}_{p}[x]$ as a product of $(n-1) / e$ irreducible polynomials $f^{\Gamma}(x)$ of degree $e$ equal to the order of $p$ in the group $\mathbb{Z}_{n}^{*} \cong C_{n-1}$. As a $G_{0}$-module, $Q$ is a direct sum of $(n-1) / e$ corresponding irreducible submodules $Q_{\Gamma}$ of dimension $e$. If $n=2$ or if $e$ is even then $-1 \in\langle p\rangle$ in $\mathbb{Z}_{n}^{*}$, so these are the $v=(n-1) / e$ irreducible summands $R_{\Delta}$ of dimension $d=e$, for $\Delta=\Gamma$, in the direct sum decomposition of $Q$ as a $G$-module. If $n>2$ and $e$ is odd then $-1 \notin\langle p\rangle$ in $\mathbb{Z}_{n}^{*}$, so we obtain $v=(n-1) / 2 e$ direct summands $R_{\Delta}=P_{\Gamma} \oplus P_{\Gamma^{*}}$ of dimension $d=2 e$ in this decomposition. In either case, there are $2^{v}-1$ maps $\mathcal{N} \in E_{p}(\mathcal{M})$, specifically $\left(\begin{array}{l}v \\ i\end{array}\right)$ with $c=\operatorname{dim}(\mathcal{N})=i d$ for each $i=1,2, \ldots, v$, affording the sum of $i$ distinct irreducible characters of degree $d$. In particular, if $n>2$ and $p \equiv \pm 1(\bmod n)$ then $d=2$, so $v=(n-1) / 2$, with the irreducible coverings affording the characters $\xi_{k}$ of $G$ for $k=1,2, \ldots,(n-1) / 2$.

(If $p=n$ then $Q \cong \mathbb{F}_{p}[x] /\left((x-1)^{n-1}\right)=\mathbb{F}_{n}[x] /\left((x-1)^{n-1}\right)$, with proper $G$-submodules of codimensions $c=1,2, \ldots, n-1$, each generated by the image of $(x-1)^{c}$. It follows that $E_{p}(\mathcal{M})$ consist of $n-1$ coverings, one for each of these dimensions $c$.)

In the next two subsections we give some simple illustrative examples.

\subsubsection{Example: $n=3$}

The first value of $n$ not covered by Sect. 8 (on the dihedron) is $n=3$. In this case the maps $\mathcal{N} \in E_{p}(\mathcal{M})$ are regular, of type $\{2 p, 3\}$ and genus $1+\frac{1}{2} p^{c-1}(p-3)$ where $c=\operatorname{dim}(\mathcal{N})$. If $p \neq 3$ then $p$ has order $e=1$ or 2 in $\mathbb{Z}_{3}^{*}$ as $p \equiv 1$ or $-1(\bmod 3)$, so as a $G_{0}$-module $Q$ is respectively a direct sum of two 1 -dimensional submodules, or is 2-dimensional and irreducible. In either case $B$ has a single orbit $\Delta \neq\{1\}$ on 
$\Omega_{3}$, namely $\left\{\zeta_{3}^{ \pm 1}\right\}$ where $\zeta_{3}$ is a primitive cube root of 1 , so $Q$ is an irreducible 2dimensional $G$-module; hence $E_{p}(\mathcal{M})$ consists of a single map $\mathcal{N}=\mathcal{M}_{0}$ of genus $(p-1)(p-2) / 2$, affording the character $\xi_{1}$ of $G$ (this is the map $\{2 p, 3\}_{6}$ in $[6$, Sect. 8.6 and Table 8]). For instance, if $p=2$ then $\mathcal{N}$ is the cube $\{4,3\}$, with $\mathcal{M}=\mathcal{N} / V_{4}$, and for $p=5,7,11$ or 13 it is the dual of the regular map R6.1, R15.2, R45.3 or R66.1 in [3].

\subsubsection{Example: $n=13$}

If $n=13$ the maps in $E_{p}(\mathcal{M})$ have type $\{2 p, 13\}$ and genus $1+\frac{1}{2} p^{c-1}(11 p-13)$. If $p= \pm 1(\bmod 13)$ then $e=1$ or 2 ; in either case $d=2$, so there are $v=12 / 2=6$ irreducible coverings, affording the characters $\xi_{1}, \ldots, \xi_{6}$, and a total of $2^{6}-1=63$ coverings $\mathcal{N} \in E_{p}(\mathcal{M})$, with $\operatorname{dim}(\mathcal{N})=2,4, \ldots, 12$. If $p \equiv 3$ or $9(\bmod 13)$ then $e=3$; this is odd, so $d=6$, and hence there are $v=2$ irreducible coverings of dimension 6; thus $E_{p}(\mathcal{M})$ consists of these two irreducible coverings and $\mathcal{M}_{0}$ of dimension 12. The same applies if $p \equiv \pm 4(\bmod 13)$, so that $d=e=6$. If $p \equiv \pm 5$ $(\bmod 13)$ then $e=4$, so $d=4$ also; thus $v=3$, so $E_{p}(\mathcal{M})$ consists of seven coverings of dimensions 4,8 and 12 . If $p \equiv \pm 2$ or $\pm 6(\bmod 13)$ then $d=e=12$, giving one irreducible covering $\mathcal{M}_{0}$ of dimension 12 .

\section{Branching over faces and vertices}

It is now easy to find the elementary abelian coverings of a Platonic map $\mathcal{M}$, where the branching is over its vertices rather than its faces: simply use the preceding results to find the corresponding coverings of the dual map $\mathcal{M}^{*}$ of $\mathcal{M}$, branched over its faces, and then take the duals of the resulting maps.

One can also use similar methods to determine the elementary abelian coverings, where the branching is over both the faces and the vertices (see also [9]). In this case, we replace $S$ with the sphere $S^{\prime}$ punctured at the set $\Phi^{\prime}=\Phi \cup \Phi^{*}$, where $\Phi$ is the set of face-centres of $\mathcal{M}$ and $\Phi^{*}$ is its set of vertices (i.e. the face-centres of $\mathcal{M}^{*}$ ). The resulting homology module $H_{1}\left(S^{\prime} ; \mathbb{F}_{p}\right)$ for $G$ on $S^{\prime}$ is the quotient $Q^{\prime}=P^{\prime} / P_{1}^{\prime}$, where $P^{\prime}$ is the permutation module $\mathbb{F}_{p} \Phi^{\prime}=\mathbb{F}_{p} \Phi \oplus \mathbb{F}_{p} \Phi^{*}$ for $G$ on $\Phi^{\prime}$, and $P_{1}^{\prime}$ is the 1-dimensional $G$-submodule of $P^{\prime}$ spanned by $\underline{\Phi}^{\prime}=\underline{\Phi}+\underline{\Phi^{*}}$. The representation and character of $G$ on $P^{\prime}$ are $\rho^{\prime}=\rho \oplus \rho^{*}$ and $\pi^{\prime}=\pi+\pi^{*}$, where $\rho$ and $\rho^{*}$ are the representations on the permutation modules $P=\mathbb{F}_{p} \Phi$ and $P^{*}=\mathbb{F}_{p} \Phi^{*}$, and $\pi$ and $\pi^{*}$ are their associated characters. Since $G$ has two orbits on $\Phi^{\prime}$, the principal character $\chi_{1}$ of $G$ has multiplicity 2 in $\pi^{\prime}$, afforded by the 1-dimensional fixed submodules $P_{1}$ and $P_{1}^{*}$ of $P$ and $P^{*}$, spanned by $\underline{\Phi}$ and $\underline{\Phi}^{*}$; deleting one copy of $\chi_{1}$ gives the character

$$
\chi^{\prime}=\pi+\pi^{*}-\chi_{1}=\chi_{1}+\chi+\chi^{*}
$$

of $G$ on $Q^{\prime}$, where $\chi=\pi-\chi_{1}$ and $\chi^{*}=\pi^{*}-\chi_{1}$ are the characters on $Q=P / P_{1}$ and $Q^{*}=P^{*} / P_{1}^{*}$. We have already determined the $G$-module structures of $P$ and of $P^{*}$ (the latter in the context of $\mathcal{M}^{*}$ rather than $\mathcal{M}$ ), so we can immediately deduce the structure of $P^{\prime}=P \oplus P^{*}$, using Lemma 2.1, and hence that of its quotient $Q^{\prime}$. 
This gives us information about the set $E_{p}^{\prime}(\mathcal{M})$ of coverings of $\mathcal{M}$ by elementary abelian $p$-groups, branched over faces and vertices, since these correspond to the proper $G$-submodules of $Q^{\prime}$.

In all such cases, $Q^{\prime}$ is an extension of a fixed $G$-submodule

$$
Q_{1}^{\prime}=\left(P_{1} \oplus P_{1}^{*}\right) / P_{1}^{\prime} \cong P_{1}\left(\cong P_{1}^{*}\right)
$$

by

$$
P /\left(P_{1} \oplus P_{1}^{*}\right)=\left(P \oplus P_{1}^{*}\right) /\left(P_{1} \oplus P_{1}^{*}\right) \oplus\left(P_{1} \oplus P^{*}\right) /\left(P_{1} \oplus P_{1}^{*}\right) \cong Q \oplus Q^{*}
$$

If the modules $P$ and $P^{*}$ both split over their fixed submodules $P_{1}$ and $P_{1}^{*}$ (equivalently, if both $f=|\Phi|$ and $f^{*}=\left|\Phi^{*}\right|$ are coprime to $p$ ), then $Q^{\prime}$ also splits over $Q_{1}^{\prime}$ : we have $P=P_{1} \oplus P^{1}$ and $P^{*}=P_{1}^{*} \oplus P^{* 1}$, so

$$
P^{\prime}=P_{1} \oplus P_{1}^{*} \oplus P^{1} \oplus P^{* 1}
$$

since $P_{1}^{\prime}$ is a submodule of $P_{1} \oplus P_{1}^{*}$ there is a complement

$$
Q^{\prime 1}=\left(P_{1}^{\prime} \oplus P^{1} \oplus P^{* 1}\right) / P_{1}^{\prime} \cong P^{1} \oplus P^{* 1} \cong Q \oplus Q^{*}
$$

for $Q_{1}^{\prime}$ on $Q^{\prime}$, giving

$$
Q^{\prime}=Q_{1}^{\prime} \oplus Q^{\prime 1} \cong P_{1} \oplus Q \oplus Q^{*}
$$

Instead of dealing with all cases in detail, we will consider one simple example, the tetrahedron, and just summarise the results in the other cases.

\subsection{The tetrahedron}

If $\mathcal{M}$ is the tetrahedron then $\mathcal{M}^{*} \cong \mathcal{M}$, and so $P \cong P^{*}$. We saw in Sect. 3 that if $p \neq 2$ then $P=P_{1} \oplus P^{1}$, affording the representation $\rho_{1} \oplus \rho_{4}$ of $G$ with character $\chi_{1}+\chi_{4}$. It follows that

$$
Q^{\prime}=Q_{1}^{\prime} \oplus Q \oplus Q^{*}
$$

affording the representation $\rho_{1} \oplus \rho_{4} \oplus \rho_{4}$ with character

$$
\chi^{\prime}=\chi_{1}+2 \chi_{4}
$$

here $Q_{1}^{\prime}$ affords $\rho_{1}$, while $Q$ and $Q^{*}$, the images in $Q^{\prime}$ of $P^{1}$ and $P^{* 1}$, both afford $\rho_{4}$. Now $Q \cong Q^{*}$, so $Q \oplus Q^{*}$ contains $p+1$ submodules $Q(\lambda) \cong Q$, where $\lambda \in \mathbb{P}^{1}(p)$ (see Sect. 2.5, and also Sect. 7 for a similar phenomenon concerning the icosahedron). Thus $Q^{\prime}$ has the following proper submodules $L$ : there is one submodule $Q \oplus Q^{*}$ of codimension $c=1$; there are $p+1$ submodules $Q_{1}^{\prime} \oplus Q(\lambda)$ of codimension 3 , and 
$p+1$ submodules $Q(\lambda)$ of codimension 4; there is one submodule $Q_{1}^{\prime}$ of codimension 6 , and one submodule 0 of codimension 7 . Thus there are $\left|E_{p}^{\prime}(\mathcal{M})\right|=2 p+5$ coverings $\mathcal{N}$ of $\mathcal{M}$, of these dimensions $c$, affording the representations $\rho_{1}, \rho_{4}, \rho_{1} \oplus \rho_{4}, \rho_{4} \oplus \rho_{4}$, and $\rho_{1} \oplus \rho_{4} \oplus \rho_{4}$ respectively. In contrast with the case of the icosahedron, orientationreversing automorphisms of $\mathcal{M}$ leave all submodules of $Q^{\prime}$ invariant, including the submodules $Q(\lambda)$, so the maps $\mathcal{N} \in E_{p}^{\prime}(\mathcal{M})$ are all regular.

The coverings of dimension 3 corresponding to $L=Q_{1}^{\prime} \oplus Q^{*}$ and $Q_{1}^{\prime} \oplus Q$ are, respectively, branched over only the faces and only the vertices; the first of these is the covering $\mathcal{M}_{0}$ of type $\{3 p, 3\}$ and genus $p^{3}-2 p^{2}+1$ described in Sect. 3 , and the second is its dual. The remaining $2 p+3$ coverings have branching of order $p^{c}-p^{c-1}$ at the four vertices and the four faces of $\mathcal{M}$, so they have type $\{3 p, 3 p\}$ and genus $3 p^{c}-4 p^{c-1}+1$. For example, when $L=Q \oplus Q^{*}$ we have a cyclic covering of genus $3(p-1)$, obtained by assigning mutually inverse monodromy permutations in $K=C_{p}$ to the vertices and face-centres of $\mathcal{M}$; for instance, if $p=3,5,7,11$ these are the maps R6.9, R12.8, R18.10, R30.10 in [3]. The $p-1$ coverings with $L=Q_{1}^{\prime} \oplus Q(\lambda)$ and $\lambda \neq 0, \infty$ have genus $3 p^{3}-4 p^{2}+1$; when $p=3$ these are the maps R46.27 of type $\{9,9\}_{4}$ and R46.28 of type $\{9,9\}_{12}$ in [3].

\subsection{The other Platonic maps}

Here we simply give the homology character $\chi^{\prime}$ for each of the remaining Platonic maps, in terms of the irreducible characters in the tables given earlier, together with a few comments. The $G$-module structure of $Q^{\prime}$ and hence the properties of the elementary abelian coverings $\mathcal{N}$ of $\mathcal{M}$ can be deduced by methods similar to those used for the tetrahedron.

If $\mathcal{M}$ is the cube or octahedron then

$$
\chi^{\prime}=\chi_{1}+\chi_{2}+\chi_{3}+\chi_{4}+2 \chi_{5} .
$$

The one-dimensional rational summands $\chi_{1}$ and $\chi_{2}$ lead to both central and non-central cyclic coverings $\mathcal{N}$ of $\mathcal{M}$. As in the case of the tetrahedron, the coefficient 2 of $\chi_{5}$ means that the number of coverings is unbounded as a function of $p$ : in fact, since $\rho_{5} \oplus \rho_{5}$ has $p+1$ submodules isomorphic to $\rho_{5}$ we have $\left|E_{p}^{\prime}(\mathcal{M})\right|=2^{4}(p+3)-1=$ $16 p+47$ for $p>3$. The resulting maps are all regular, since all $G$-submodules of $Q$ and $Q^{*}$, and hence of $Q^{\prime}$, are invariant under orientation-reversing automorphisms of $\mathcal{M}$ (see Sects. 4, 5).

If $\mathcal{M}$ is the dodecahedron or icosahedron then

$$
\chi^{\prime}=\chi_{1}+2 \chi_{2}+2 \chi_{3}+2 \chi_{4}+2 \chi_{5}
$$

The character $\chi_{1}$ again leads to central cyclic coverings, but now there are no noncentral cyclic coverings. The characters $\chi_{4}$ and $\chi_{5}$ are both rational, so the representations $\rho_{4}$ and $\rho_{5}$ are realised over $\mathbb{F}_{p}$, as are $\rho_{2}$ and $\rho_{3}$ if $p \equiv \pm 1(\bmod 5)$; in this case counting proper $G$-submodules gives $\left|E_{p}^{\prime}(\mathcal{M})\right|=2(p+3)^{4}-1$. If $p \equiv \pm 2$ $(\bmod 5)$, however, we obtain a 6-dimensional irreducible representation over $\mathbb{F}_{p}$ with 
character $\chi_{2}+\chi_{3}$ and multiplicity 2 , so that $\left|E_{p}^{\prime}(\mathcal{M})\right|=2(p+3)^{3}-1$. In either case the summand $2 \chi_{4}$ of $\chi^{\prime}$ is afforded by an 8-dimensional submodule $Q_{a, b}$ (defined in Sect. 7), inherited from the action of $G$ on the faces of an icosahedron, or equivalently the vertices of a dodecahedron; since the antipodal automorphism of $\mathcal{M}$ acts non-trivially on the 4-dimensional submodules $Q(\lambda)$ of $Q_{a, b}$, we see that $E_{p}^{\prime}(\mathcal{M})$ contains $(p+3)^{3}(p-1)$ or $(p+3)^{2}(p-1)$ chiral pairs of maps as $p \equiv \pm 1$ or \pm 2 $(\bmod 5)$.

If $\mathcal{M}$ is the dihedron $\{n, 2\}$ or the hosohedron $\{2, n\}$ then

$$
\chi^{\prime}=\chi_{1}+\chi_{2}+\sum_{k=1}^{(n-1) / 2} \xi_{k}
$$

if $n$ is odd, and

$$
\chi^{\prime}=\chi_{1}+\chi_{2}+\chi_{3}+\sum_{k=1}^{(n-2) / 2} \xi_{k}
$$

if $n$ is even. The 1 -dimensional rational characters $\chi_{k}$ give rise to cyclic coverings, central when $k=1$ and non-central when $k>1$. The fact that $\chi^{\prime}$ is multiplicityfree means that $\left|E_{p}^{\prime}(\mathcal{M})\right|$ is bounded above as a function of $p$, by $2^{(n+3) / 2}-1$ or $2^{(n+4) / 2}-1$ as $n$ is odd or even. These bounds are attained when $p \equiv 1(\bmod n)$, so that the representations corresponding to the characters $\xi_{k}$ all reduce $\bmod p$ to 2dimensional representations over $\mathbb{F}_{p}$. For each $n$ there are infinitely many such primes $p$, by Dirichlet's theorem on primes in arithmetic progressions. As in Sect. 8 and Sect. 9, the maps in $E_{p}^{\prime}(\mathcal{M})$ are regular for all $p$.

\section{Branching over edges}

Hypermaps (Grothendieck's dessins d'enfants, see [8,12]) are generalisations of maps, corresponding to triangle groups $\Delta(p, q, r)$ with $q$ not necessarily equal to 2 . These arise if we extend the investigation of coverings of the Platonic maps to allow branching over their edges. The general principles are the same as for branching over the faces, except that $\Phi$ is now replaced with the set $\Phi^{\dagger}$ of edges of $\mathcal{M}$, the sphere is punctured at their midpoints, and $H$ is the subgroup $\langle y\rangle \cong C_{2}$ of $G$ leaving invariant an edge. Dual pairs of maps $\mathcal{M}$ and $\mathcal{M}^{*}$ can be treated together, since duality induces $G$ isomorphisms between their edge sets. As in Sect. 10, we will study the resulting set $E_{p}^{\dagger}(\mathcal{M})$ of coverings of $\mathcal{M}$ by concentrating on the tetrahedron as a typical example. The decompositions of the character $\chi^{\dagger}$ of $G$ on the homology module are as follows, with the notation for irreducible characters as before. For the tetrahedron,

$$
\chi^{\dagger}=\chi_{2}+\chi_{3}+\chi_{4} .
$$


For the cube and the octahedron,

$$
\chi^{\dagger}=\chi_{3}+2 \chi_{4}+\chi_{5}
$$

For the dodecahedron and the icosahedron,

$$
\chi^{\dagger}=\chi_{2}+\chi_{3}+2 \chi_{4}+3 \chi_{5}
$$

For the dihedron and the hosohedron, if $n$ is odd then

$$
\chi^{\dagger}=\sum_{k=1}^{(n-1) / 2} \xi_{k},
$$

the same decomposition as for branching over the faces of the hosohedron, by the duality between edges and faces of this map; if $n$ is even then taking $H=\langle a b\rangle$ as the subgroup stabilising an edge (since we took $\langle b\rangle$ in Sect. 9.1 as the stabiliser of a face), we have

$$
\chi^{\dagger}=\chi_{1}+\chi_{4}+\sum_{k=1}^{(n-2) / 2} \xi_{k} .
$$

\subsection{The tetrahedron}

Let $\mathcal{M}$ be the tetrahedron, so that the coverings $\mathcal{N}$ are hypermaps of type $(3,2 p, 3)$ and genus $2 p^{c}-3 p^{c-1}+1$. If $p \equiv 1(\bmod 3)$ then $\chi^{\dagger}$ decomposes over $\mathbb{F}_{p}$ as above, with irreducible summands $\chi_{2}, \chi_{3}$ and $\chi_{4}$ of degrees 1,1 and 3 , so we obtain seven coverings $\mathcal{N}$, with $c=1,1,2,3,4,4$ and 5. Since $\chi_{2}$ and $\chi_{3}$ are transposed by orientation-reversing automorphisms of $\mathcal{M}$, those with $c=1$ or 4 occur in chiral pairs, whereas the other three are regular. For instance, if $p=7$ then the two cyclic coverings (with $c=1$ ) are duals of the chiral pair of hypermaps CH12.1 of genus 12 and type $(3,3,14)$ in [3]. If $2<p \equiv 2(\bmod 3)$ then $\chi^{\dagger}$ decomposes as a sum of two irreducible characters $\chi_{2}+\chi_{3}$ and $\chi_{4}$ of degrees 2 and 3, so in this case there are three coverings $\mathcal{N}$ with $c=2,3$ and 5, all of them regular hypermaps. For instance, if $p=5$ then the covering with $c=2$ is a dual of the hypermap RPH36.3 in [3].

\subsection{The other Platonic maps}

In the cases of the cube and the octahedron, there is one irreducible covering with $c=2$, affording $\rho_{3}$, and there are $p+2$ with $c=3$, of which $p+1$ afford $\rho_{4}$ and one affords $\rho_{5}$; thus $\left|E_{p}^{\dagger}(\mathcal{M})\right|=4 p+11$ and there are no cyclic coverings. Since $G$ has rank 7 as a permutation group on $\Phi^{\dagger}$, whereas $A$ has rank 5, some of the hypermaps in $E_{p}^{\dagger}(\mathcal{M})$ are chiral; in fact, two of the irreducible $G$-submodules of $Q^{\dagger}$ affording $\rho_{4}$ are non-isomorphic $A$-submodules, while the other $p-1$ are transposed in pairs by 
orientation-reversing automorphisms, so there are 15 regular hypermaps in $E_{p}^{\dagger}(\mathcal{M})$ and $2(p-1)$ chiral pairs.

If $\mathcal{M}$ is the dodecahedron or the icosahedron, then the presence of the conjugate pair of summands $\chi_{2}$ and $\chi_{3}$ in $\chi^{\dagger}$ means that the decomposition of $Q^{\dagger}$, and hence the number and nature of the coverings, again depends on whether $p \equiv \pm 1$ or \pm 2 $(\bmod 5)$, as in Sects. 6, 7 and 10.2. As in the case of the cube and the octahedron, there are chiral pairs but no cyclic coverings.

Hypermap coverings branched over the edges of a hosohedron $\mathcal{M}=\{2, n\}$ (or its dual dihedron) can be dealt with as in Sect. 9 by considering the map coverings branched over the faces of the hosohedron obtained by rotating $\mathcal{M}$ through $\pi / n$ about its two vertices.

\section{Branching over vertices, edges and faces}

Just as we considered branching over the vertices and faces in Sect. 10, one can also consider coverings which are branched over any combination of vertices, edges and faces. In the most general case, where we allow branching over all three sets, $G$ has three orbits on the punctures of the sphere; the permutation module $P^{\prime \prime}$ is therefore the direct sum of $P, P^{*}$ and a third permutation module $P^{\dagger}$ with $\Phi^{\dagger}$ as its basis, and the corresponding homology character is

$$
\chi^{\prime \prime}=\pi+\pi^{*}+\pi^{\dagger}-\chi_{1}=2 \chi_{1}+\chi+\chi^{*}+\chi^{\dagger} .
$$

The corresponding coverings of each $\mathcal{M}$ can now be found by using the techniques described earlier. For instance, in all cases the character $\chi_{1}$ has multiplicity 2 in $\chi^{\prime \prime}$, yielding $p+1$ central cyclic coverings of $\mathcal{M}$ for each prime $p$ not dividing $|G|$ : three are branched over just two from the three sets of vertices, edges and faces, and $p-2$ are branched over all three sets. These are all quotients of a 2-dimensional central covering.

\subsection{The tetrahedron}

Taking the tetrahedron again as a typical example, we have

$$
\chi^{\prime \prime}=2 \chi_{1}+\chi_{2}+\chi_{3}+3 \chi_{4}
$$

If $p \equiv 1(\bmod 3)$ then this gives the direct sum decomposition of $Q^{\prime \prime}$, with the principal submodule of multiplicity 2 , two conjugate non-principal 1-dimensional submodules of multiplicity 1 , and a 3-dimensional irreducible submodule of multiplicity 3. Counting proper submodules, we find $8(p+3)\left(p^{2}+p+2\right)-1$ coverings, of which $p+1$ are central and cyclic, and two are non-central and cyclic. As in Sect. 11.1, the appearance of the characters $\chi_{2}$ and $\chi_{3}$, transposed by orientation-reversing automorphisms, gives rise to chiral hypermaps, this time forming $2(p+3)\left(p^{2}+p+2\right)$ pairs. 
Even in the smallest case, when $p=7$, there are far too many coverings to describe them all, but we can at least describe the central cyclic coverings. Branching over edges and either faces or vertices yields the regular hypermap RPH24.3 in [3], of genus 24 and type $(3,14,21)$, or its dual of type $(21,14,3)$; in each case the same monodromy element of $K=C_{7}$ is assigned to each of the ten branch-points. Branching over the vertices and faces yields the regular map R18.10, as in Sect. 10.1. Branching over the vertices, edges and faces yields five regular hypermaps of genus 36 and type $(21,14,21)$; these are duals of RPH36.62, RPH36.63 and RPH36.64. The two noncentral cyclic coverings form a chiral pair of genus 12, branched over the edges and described in Sect. 11.1.

If $2<p \equiv 2(\bmod 3)$ then in the above decomposition of $Q^{\prime \prime}$ the two non-principal 1-dimensional summands are replaced with one irreducible summand of dimension 2 , which affords the character $\chi_{2}+\chi_{3}$. In this case there are $4(p+3)\left(p^{2}+p+2\right)-1$ coverings in $E_{p}^{\prime \prime}(\mathcal{M})$; the $p+1$ cyclic coverings are all central, and there are no chiral pairs.

If $p=5$, for instance, the cyclic coverings are as follows. Branching over edges and either faces or vertices yields the regular hypermap RPH16.8, of genus 16 and type $(3,10,15)$, or its dual of type $(15,10,3)$; in each case the same monodromy element of $K=C_{5}$ is assigned to each of the ten branch-points. Branching over the vertices and faces yields the regular map R12.8, as in Sect. 10.1. Branching over the vertices, edges and faces yields three regular hypermaps of genus 24 and type $(15,10,15)$; these are duals of RPH24.37 and RPH24.38.

Acknowledgments The author is grateful to Young Soo Kwon for raising this problem, Marston Conder and Jicheng Ma for useful information on their work in progress on graph coverings, Rubén Hidalgo and Roman Nedela for helpful comments on an earlier draft of this paper, and an anonymous referee for some very useful advice on improving its presentation.

\section{References}

1. Accola, R.D.M.: On the number of automorphisms of a closed Riemann surface. Trans. Am. Math. Soc. 131, 398-408 (1968)

2. Cohen, J.M.: On Hurwitz extensions by PSL 2 (7). Math. Proc. Camb. Philos. Soc. 86, 395-400 (1979)

3. Conder, M.D.E.: Regular maps and hypermaps of Euler characteristic -1 to -200 . J. Comb. Theory Ser. B 99, 455-459 (2009). Associated lists of computational data from http://www.math.auckland.ac. nz/conder/hypermaps.html.

4. Conder, M.D.E., Ma, J.: Arc-transitive abelian regular covers of cubic graphs. J. Algebra 387, 215-242 (2013)

5. Conder, M.D.E., Ma, J.: Arc-transitive abelian regular covers of the Heawood graph. J. Algebra 387, 243-267 (2013)

6. Coxeter, H.S.M., Moser, W.O.J.: Generators and Relations for Discrete Groups, 4th edn. SpringerVerlag, Berlin, Heidelberg, New York (1980)

7. Farkas, H.M., Kra, I.: Riemann Surfaces. Springer-Verlag, New York, Berlin, Heidelberg (1991)

8. Grothendieck, A.: Esquisse d'un Programme. In: Lochak, P., Schneps, L. (eds.) Geometric Galois Actions 1. Around Grothendieck's Esquisse d'un Programme. London Mathematical Society Lecture Note Series, vol. 242, pp. 5-84. Cambridge University Press, Cambridge (1997)

9. Hu, K.: Group Extensions and Coverings of Maps. Ph.D. thesis, Matej Bel University, Banská Bystrica, Slovakia (2013)

10. Hu, K., Nedela, R., Wang, N.-E.: Branched cyclic regular coverings over platonic maps. Eur. J. Comb. 36, 531-549 (2014) 
11. Jones, G.A., Singerman, D.: Theory of maps on orientable surfaces. Proc. Lond. Math. Soc. 3(37), 273-307 (1978)

12. Jones, G.A., Singerman, D.: Belyŭ functions, hypermaps and Galois groups. Bull. Lond. Math. Soc. 28, 561-590 (1996)

13. Jones, G.A., Surowski, D.B.: Regular cyclic coverings of the Platonic maps. Eur. J. Comb. 21, 333-345 (2000)

14. Kazaz, M.: Finite Groups and Surface Coverings. Ph.D. thesis, University of Southampton, Southampton (1997)

15. Kazaz, M.: Homology action on regular hypermaps of genus 2. Commun. Fac. Sci. Univ. Ank. Sér. A1 Math. Stat. 51, 1-18 (2002)

16. Kazaz, M.: Elementary abelian coverings of regular hypermaps of types $(5,5,5)$ and $(5,2,10)$ of genus 2. Proc. Jangjeon Math. Soc. 12, 189-202 (2009)

17. Kwak, J.H., Oh, J.-M.: Arc-transitive elementary abelian covers of the octahedron graph. Linear Algebra Appl. 429, 2180-2198 (2008)

18. Maclachlan, C.: A bound for the number of automorphisms of a compact Riemann surface. J. Lond. Math. Soc. 44, 265-272 (1969)

19. Malnič, A., Marušič, D., Potočnik, P.: Elementary abelian covers of graphs. J. Algebr. Combin. 20, 71-97 (2004)

20. Surowski, D.B., Jones, G.A.: Cohomological constructions of regular cyclic coverings of the Platonic maps. Eur. J. Combin. 21, 407-418 (2000) 